\title{
EVOLUTIONARY PRISONER'S DILEMMA GAMES WITH ONE-DIMENSIONAL LOCAL INTERACTION AND IMITATION
}

\author{
HSIAO-CHI CHEN, ${ }^{*}$ National Taipei University \\ YUNSHYONG CHOW, ${ }^{* *}$ Academia Sinica
}

\begin{abstract}
In this paper we explore the impact of imitation rules on players' long-run behaviors in evolutionary prisoner's dilemma games. All players sit sequentially and equally spaced around a circle. Players are assumed to interact only with their neighbors, and to imitate either their successful neighbors and/or themselves or the successful actions taken by their neighbors and/or themselves. In the imitating-successful-player dynamics, full defection is the unique long-run equilibrium as the probability of players' experimentations (or mutations) tend to 0 . By contrast, full cooperation could emerge in the long run under the imitating-successful-action dynamics. Moreover, it is discovered that the convergence rate to equilibrium under local interaction could be slower than that under global interaction.
\end{abstract}

Keywords: Cooperation; imitation; local interaction; long-run equilibrium; prisoner's dilemma game

2000 Mathematics Subject Classification: Primary 91A22; 60J20

\section{Introduction}

Whether (full) cooperation can be sustained in repeatedly played prisoner's dilemma (PD) games interests many researchers. Several approaches have been explored to achieve the cooperation goal. The folk theorem, assuming that players are longsightedly rational, has been shown to be effective in this subject. More precisely, the folk theorem shows that cooperative payoff could be achieved by some equilibrium if players are sufficiently patient. Evolutionary games with boundedly rational players are promising approaches as well. Nevertheless, whether players will eventually cooperate would depend on how to set players' boundedly rational behaviors. For instance, through the aspiration-level adjustment over periods based on realized payoffs, Karandikar et al. (1998) and Palomino and Vega-Redondo (1999) showed that cooperation is possible in the long run among two players and a continuum of players, respectively. In the replicator dynamics, Bergstrom and Stark (1993), Nowak and Sigmund (1995), and Wiseman and Yilankaya (2001) proved that cooperation among a continuum of players could emerge in the long run.

Oppositely, cooperation is impossible under the Darwinian-type dynamics studied in Kandori et al. (1993) and Robson and Vega-Redondo (1996). In Kandori et al. (1993) individuals play with all other players, while players are randomly paired to play the game multiple rounds per

Received 30 May 2008; revision received 26 October 2008.

* Postal address: Department of Economics, National Taipei University, 151, University Road, San-Shia, Taipei County 23741, Taiwan, R. O. C. Email address: hchen@mail.ntpu.edu.tw

** Postal address: Institute of Mathematics, Academia Sinica, Taipei, Taiwan 115, R. O. C.

Email address: chow@math.sinica.edu.tw 
period in Robson and Vega-Redondo (1996). A detailed comparison between Kandori et al. (1993) and Robson and Vega-Redondo (1996) can be found in Miekisz (2008). Cooperation cannot be achieved either under the best-response dynamics studied in Ellison (1993), in which individuals interact only with their neighbors. However, combining an imitating-successfulaction mechanism with local interaction, Eshel et al. (1998) showed that only the states with cooperators being the majority could survive and be the long-run equilibria (LRE) for a large population size. Nevertheless, their work does not tell us exactly which states, especially the full-cooperation state, are LRE.

The first purpose of this paper is to answer the questions that remain in Eshel et al. (1998) by deriving all the LRE and the convergence rates to these equilibria. In our model, players meet with each of their two neighbors once to play the PD game at each time period. Players are assumed to imitate the successful actions yielding the higher average payoffs. After completing the strategy revision, players will independently experiment with new strategies with a small probability. Under our model, which states are LRE and the convergence rates to these equilibria depend on the game's payoff structure and the population size. For a large population size and cooperative payoff, full cooperation would emerge in the long run with a less-than-one positive probability. Moreover, the states with cooperators being the majority are not necessarily the LRE. It is also discovered that players could move slower to full defection under local interaction than under global interaction. This outcome is different from that of Ellison (1993), who discovered that the convergence rate to the long-run equilibrium under local interaction is faster than that under global interaction in the best-reply dynamics.

Eshel et al. (1998) demonstrated that players' local interaction settings are necessary for the emergence of cooperation under a specific imitation mechanism. However, one may wonder whether the local interaction setting is also necessary under other imitation mechanisms. Thus, the second purpose of this paper is to investigate this issue by changing players' imitation rules. Unlike in Eshel et al. (1998), we presume that players imitate the successful neighbors or themselves earning the highest total payoffs. It is found that full defection is the unique longrun equilibrium. Moreover, the convergence rate to full defection is independent of the game's payoff structure and population size. Hence, our results show that, for large cooperative payoffs, players' imitation rules are critical in determining their long-run behavior in evolutionary PD games, in addition to the local interaction setting.

The intuition behind different outcomes under the two imitation rules mentioned above is as follows. The force deviating from cooperation could be weakened in the process of averaging action payoffs under the imitating-successful-action rule, but that force remains dominant under the imitating-successful-player rule. Thus, full cooperation might flourish under the former rule.

The above strategy revision dynamics without mutation can be described as a Markov chain with the strategy profiles of all players as states. Each state is either transient or lies in a closed connected component and is thus called a stationary state. In particular, full cooperation and full defection are two typical absorbing states and are thus stationary states. The introduction of a mutation mechanism changes the landscape of the states. Each stationary state is now associated with a basin. The deeper the basin is, the longer the mutated process will stay in it. In other words, the LRE desired are those stationary states which have the deepest basin. Though complicated, computation of the basin depth can be done via the method of Freidlin and Wentzell (1984). It turns out that, under the imitating-successful-action dynamics, the set of LRE and the convergence rate to equilibrium depend on the population size and on the payoff structure as well. 
The rest of the paper is organized as follows. Two imitation rules are presented in Section 2. The associated results are demonstrated in Section 3. The method of Freidlin and Wentzell is briefly introduced in Section 4, and the proofs of our findings are given in Section 5.

\section{The model}

Our model is described as follows. Let $N=\{1,2, \ldots, n\}, n \geq 5$, be the set of players. These players are assumed to sit sequentially and equally spaced around a circle. Each player has exactly two neighbors. For $i \in N$, let $N_{i}=\{i-1, i+1\}$ be the set of player $i$ 's neighbors. Note that player $n+i$ is the same as player $i$ by modulo $n$. At time period $t \in\{0,1,2, \ldots\}$, players meet with each of their two neighbors once to play the following PD game:

\begin{tabular}{c|c|c|}
\multicolumn{1}{c}{$D$} & \multicolumn{1}{c}{$C$} \\
\cline { 2 - 3 }$D$ & $a, a$ & $b, c$ \\
\cline { 2 - 3 }$C$ & $c, b$ & $d, d$ \\
\cline { 2 - 3 } & &
\end{tabular}

Here $\{C, D\}$ is the strategy set for all players. By convention, $C$ and $D$ represent the cooperation and defection (or noncooperation) strategies, respectively. Moreover,

$$
a, b, c, \text { and } d \text { are payoffs with } b>d>a \geq c .
$$

When $a>c$, strategy $D$ strictly dominates strategy $C$ and $(D, D)$ is the unique Nash equilibrium. When $a=c$, strategy $D$ weakly dominates strategy $C$, and $(D, D),(C, D)$, and $(D, C)$ are all Nash equilibria. Nevertheless, $(C, C)$ yields higher payoff than $(D, D)$ in both cases above. Accordingly, the state space $S$ of our dynamic systems is the set of all players' strategy profiles. That is, $S=\{C, D\}^{n}$ with element $s=\left(s_{1}, s_{2}, \ldots, s_{n}\right)$, where $s_{i}$ is the strategy adopted by player $i \in N$. For simplicity, label by $\boldsymbol{C}=(C, C, \ldots, C)$ and $\boldsymbol{D}=(D, D, \ldots, D)$ the states where all players cooperate and defect, respectively. In the beginning of each period, players' actions and payoffs that occurred (after mutation) in the last period are observable to their neighbors.

Our local-interaction imitation dynamic system consists of two successive parts: imitation and mutation. In the imitation process, two rules are considered. In the first rule, each player $i$ is assumed to imitate the most successful player, i.e. the player earning the highest total payoff among his neighbors and himself. More precisely, given state $s=\left(s_{1}, s_{2}, \ldots, s_{n}\right) \in S$ at time $t$, let $z_{i}(s)$ represent player $i$ 's total payoff after playing with each of his neighbors once at time $t$. It is easy to see that

$$
z_{i}(\boldsymbol{s})= \begin{cases}b n_{i}^{C}(\boldsymbol{s})+a\left(2-n_{i}^{C}(\boldsymbol{s})\right) & \text { if } s_{i}=D, \text { i.e. player } i \text { chooses strategy } D, \\ d n_{i}^{C}(\boldsymbol{s})+c\left(2-n_{i}^{C}(\boldsymbol{s})\right) & \text { if } s_{i}=C, \text { i.e. player } i \text { chooses strategy } C .\end{cases}
$$

Here $n_{i}^{C}(s)=\left|\left\{j \in N_{i}: s_{j}=C\right\}\right|$ is the number of player $i$ 's neighbors choosing strategy $C$ at time $t$. Accordingly, player $i$ 's next-period rational choice $r_{i}(s)$ satisfies

$$
r_{i}(\boldsymbol{s}) \in M_{i}(\boldsymbol{s}):=\left\{s_{j}: z_{j}(\boldsymbol{s})=\max z_{k}(\boldsymbol{s}) \text { for } k \in N_{i} \cup\{i\}\right\} .
$$

In the second rule, after playing with his neighbors, each player $i$ is assumed to imitate the most successful action, i.e. the action yielding the highest average payoff which was adopted 
among his neighbors and himself at time $t$. Let $\delta$ be the Kronecker notation. Then

$$
a_{i}^{E}(s)= \begin{cases}\frac{\sum_{k \in N_{i} \cup\{i\}} z_{k}(s) \delta_{E, s_{k}}}{\sum_{k \in N_{i} \cup\{i\}} \delta_{E, s_{k}}} & \text { if } E \in\left\{s_{i-1}, s_{i}, s_{i+1}\right\}, \\ -\infty & \text { if } E \neq s_{i-1}=s_{i}=s_{i+1},\end{cases}
$$

represents the average payoff for strategy $E \in\{C, D\}$ among player $i$ and his neighbors. Therefore, player $i$ 's next-period rational choice $r_{i}(s)$ satisfies

$$
r_{i}(s) \in \bar{M}_{i}(s):=\left\{E \in\{C, D\}: a_{i}^{E}(s)=\max \left(a_{i}^{C}(s), a_{i}^{D}(s)\right)\right\} .
$$

The computations of $M_{i}(s)$ in (2.3) and $\bar{M}_{i}(s)$ in (2.5) involve the strategies taken by five consecutive players from $i-2$ to $i+2$. There are 32 cases in total to be considered in order to determine $r_{i}(s)$ from (2.3) or (2.5). Since

$$
r_{i}(s)=s_{i} \quad \text { if } s_{i-1}=s_{i}=s_{i+1},
$$

14 cases are left by symmetry. These 14 cases can be classified into four categories: (i) only player $i$ in $N_{i} \cup\{i\}$ chooses strategy $D$, (ii) only player $i$ in $N_{i} \cup\{i\}$ chooses strategy $C$, (iii) two players including player $i$ in $N_{i} \cup\{i\}$ choose strategy $D$, and (iv) two players including player $i$ in $N_{i} \cup\{i\}$ choose strategy $C$. The corresponding values of $a_{i}^{C}(\boldsymbol{s}), a_{i}^{D}(\boldsymbol{s})$, and $z_{j}(\boldsymbol{s})$ for $j \in N_{i} \cup\{i\}$ are presented in Tables 1, 2, 3, and 4 in Appendix A. For example, if $\left(s_{i-2}, s_{i-1}, s_{i}, s_{i+1}, s_{i+2}\right)=$ $(D, D, D, C, D)$ then $z_{i-1}(\boldsymbol{s})=a+a=2 a, z_{i}(\boldsymbol{s})=a+b$, and $z_{i+1}(\boldsymbol{s})=c+c=2 c$. Since $s_{i-1}=s_{i}=D$ and $s_{i+1}=C$, we obtain, by (2.4), that $a_{i}^{D}(\boldsymbol{s})=\left(z_{i-1}(s)+z_{i}(s)\right) / 2=$ $(3 a+b) / 2$ and $a_{i}^{C}(s)=z_{i+1}(s)=2 c$.

Some ambiguity occurs when $M_{i}(s)=\{C, D\}$ or $\bar{M}_{i}(s)=\{C, D\}$. For simplicity, we require in this paper that, by inertia, player $i$ always sticks to his original strategy $s_{i}$ at time $t$ whenever ambiguity occurs. Under this strict rule,

$$
r_{i}(s)=s_{i} \quad \text { if and only if } \quad s_{i} \in M_{i}(s)\left(\text { or } s_{i} \in \bar{M}_{i}(s)\right) .
$$

The loose rule that both $\{C, D\}$ can be chosen as the rational choice $r_{i}(s)$ by setting

$$
\mathrm{P}\left(r_{i}(s)=C\right)=\mathrm{P}\left(r_{i}(s)=D\right)=\frac{1}{2}
$$

can be dealt with similarly, and the same results hold with slight modifications.

The above imitation process induces a time-homogeneous Markov chain on $S$ with its probability transition matrix $Q_{0}: S \times S \rightarrow[0,1]$ given by

$$
Q_{0}(\boldsymbol{s}, \boldsymbol{u})=1 \text { or } 0 \text { depending on whether } \boldsymbol{u}=\boldsymbol{r}(\boldsymbol{s}) \text { or not, respectively, }
$$

where $\boldsymbol{r}(\boldsymbol{s})=\left(r_{1}(\boldsymbol{s}), r_{2}(\boldsymbol{s}), \ldots, r_{n}(\boldsymbol{s})\right)$ is uniquely determined for state $\boldsymbol{s} \in S$ by (2.7). In particular, from (2.6) we have $\boldsymbol{r}(\boldsymbol{C})=\boldsymbol{C}$ and $\boldsymbol{r}(\boldsymbol{D})=\boldsymbol{D}$. Hence,

$$
Q_{0}(\boldsymbol{C}, \boldsymbol{C})=Q_{0}(\boldsymbol{D}, \boldsymbol{D})=1 .
$$

In the Markov chain terminology, each state in $S$ is either transient or lies in a closed connected component $\mathcal{F}$. In the latter case, it is called a stationary state. By definition, all states in any $\mathcal{F}$ can reach each other. A state is called absorbing if and only if it lies in some $\mathcal{F}$ with $|\mathcal{F}|=1$. 
In the literature, $\mathcal{F}$ is sometimes called an absorbing set. Owing to $|S|<\infty$, the set $S_{0}$ of all stationary states is nonempty and can be characterized by

$$
S_{0}=\left\{s \in S: \lim _{t \rightarrow \infty}\left(v Q_{0}^{t}\right)(s)>0 \text { for some initial distribution } v \text { on } S\right\} .
$$

Since $\boldsymbol{C}$ and $\boldsymbol{D}$ are absorbing states under $Q_{0}$ by (2.9), we have

$$
\{\boldsymbol{C}, \boldsymbol{D}\} \subseteq S_{0} .
$$

After completing the imitation process, players will simultaneously, but independently, alter their rational choices $\left\{r_{i}(s)\right\}$ with identical probability $\varepsilon>0$, which is called the mutation rate and can be regarded as the probability of players' experimenting with new strategies. Altogether, our local-interaction imitation dynamics define a Markov chain $\left\{X_{t}: t=0,1, \ldots\right\}$ on $S$ with its probability transition matrix $Q_{\varepsilon}$, a perturbation of $Q_{0}$ in (2.8), given by

$$
Q_{\varepsilon}(\boldsymbol{s}, \boldsymbol{u})=\varepsilon^{d(\boldsymbol{r}(\boldsymbol{s}), \boldsymbol{u})}(1-\varepsilon)^{n-d(\boldsymbol{r}(\boldsymbol{s}), \boldsymbol{u})} \quad \text { for all } \boldsymbol{s}, \boldsymbol{u} \in S,
$$

where $d(\boldsymbol{r}(\boldsymbol{s}), \boldsymbol{u})=\left|\left\{i \in N: r_{i}(\boldsymbol{s}) \neq u_{i}\right\}\right|$ is the number of mismatches between the next truly adopted strategy $\boldsymbol{u}$ and the revised rational choice $\boldsymbol{r}(\boldsymbol{s})$ at state $\boldsymbol{s}$.

Because $Q_{\varepsilon}(\boldsymbol{s}, \boldsymbol{u})>0$ for all $\boldsymbol{s}, \boldsymbol{u} \in S$, the mutation mechanism makes our dynamic process $\left\{X_{t}\right\}$ ergodic. Let $\mu_{\varepsilon}$ be the associated unique invariant distribution, which is independent of the initial distribution and characterized by

$$
\mu_{\varepsilon}=\mu_{\varepsilon} Q_{\varepsilon} .
$$

We are interested in the limit probability distribution $\mu_{*}:=\lim _{\varepsilon \rightarrow 0} \mu_{\varepsilon}$, whose existence is shown in Section 5, and, in particular, whether

$$
C \in S_{*}:=\left\{s \in S: \mu_{*}(s)>0\right\},
$$

which means that full cooperation among players is possible in the long run. Elements in $S_{*}$ are called the stochastically stable states or LRE. Moreover, we are interested in the order estimate of $\mathrm{E}_{\varepsilon}(T)$, where

$$
T=\inf \left\{t \geq 0: X_{t} \in S_{*}\right\}
$$

is the first time that $\left\{X_{t}\right\}$ hits $S_{*}$ with, say, the initial $X_{0}$ uniformly distributed on $S$.

Letting $\varepsilon \downarrow 0$ in (2.13), Vega-Redondo (2003, pp. 477-479) showed that $\mu_{*}=\mu_{*} Q_{0}$ and, thus, by (2.10),

$$
S_{*} \subseteq S_{0}
$$

As will be shown in Section 5, we will characterize $S_{0}$ first. Using the method of Freidlin and Wentzell (1984), we are then able to find $S_{*}$ and the order estimate for $\mathrm{E}_{\varepsilon}(T)$.

\section{The results}

In order to specify $S_{0}$, we first introduce some notation. Any state in the set

$$
M:=S_{0} \backslash\{\boldsymbol{C}, \boldsymbol{D}\}
$$

is called a mixed stationary state, which means that cooperators and defectors coexist peacefully. If $M \neq \varnothing$, any $s \in M$ consists of some $D$-strings alternating with an equal number of $C$-strings as follows:

$$
\cdots \underbrace{D \cdots D}_{d_{k}} \underbrace{C \cdots C}_{c_{k}} \underbrace{D \cdots D}_{d_{1}} \underbrace{C \cdots C}_{c_{1}} \underbrace{D \cdots D}_{d_{2}} \underbrace{C \cdots C}_{c_{2}} \cdots .
$$


Here $d_{i}$ and $c_{j}$ are the respective lengths of the $i$ th $D$-string and the $j$ th $C$-string starting from a certain player. Note that the number of $D$-strings in (3.2) equals the number of $C$-strings as players sit around a circle. For positive integers $m$ and $\ell$, define the sets

$$
\begin{gathered}
M_{\geq m, \geq \ell}:=\left\{s \in S: \text { all } d_{i} \geq m \text { and } c_{j} \geq \ell \text { in (3.2) }\right\} \\
\text { and } M_{m, \ell}:=\left\{s \in S: \text { all } d_{i}=m \text { and } c_{j}=\ell \text { in (3.2) }\right\} .
\end{gathered}
$$

Remember that throughout the paper we assume that $|N|=n \geq 5$ and $b>d>a \geq c$ by (2.1). In particular, we have $a+b>c+d$. The notation $f(\varepsilon) \approx \varepsilon^{\alpha}$ means that $\lim _{\varepsilon \downarrow 0} f(\varepsilon) / \varepsilon^{\alpha}$ exists and is positive.

Results for the imitation of the most successful player and action dynamics are presented in Theorems 3.1 and 3.2, below, respectively. The proofs are given in Section 5.

Theorem 3.1. Assume that (2.1), the imitation of the most successful player dynamics, (2.3), and the strict rule (2.7) hold. Then $S_{*}=\{\boldsymbol{D}\}$ and $\mathrm{E}_{\varepsilon}(T) \approx \varepsilon^{-1}$ as $\varepsilon \downarrow 0$. Moreover,

(i) if $a+b>2 d$ then $S_{0}=\{\boldsymbol{C}, \boldsymbol{D}\}$; and

(ii) if $a+b \leq 2 d$ then $S_{0}=\{\boldsymbol{C}, \boldsymbol{D}\} \cup M_{\geq 2, \geq 3}$.

Theorem 3.1 demonstrates that full defection, $\boldsymbol{D}$, is the unique long-run equilibrium of the imitation of the most successful player dynamics, though $S_{0}$ depends on whether $a+b \leq 2 d$ or not. In the former case, Theorem 3.1(ii) shows that $M$ defined in (3.1) is nonempty. Roughly speaking, the basin of attraction at state $\boldsymbol{D}$ is the largest one for the process $\left\{X_{t}\right\}$ and, thus, $\boldsymbol{D}$ becomes the unique long-run equilibrium as $\varepsilon \rightarrow 0$. Theorem 3.1 also shows that the convergence rate to $\boldsymbol{D}$ has order $\varepsilon^{-1}$, which is independent of the game's payoff structure and population size.

Imitation of the most successful player dynamics without mutation has been used in Nowak and May (1992), (1993), Nowak et al. (1994), and Outkin (2003) to study evolutionary PD games on general spatial structures, such as two-dimensional lattices and the present onedimensional circle. Assuming that $a=c=0, d=1$, and $2>b>1$, they showed that the set $M$ of mixed stationary states is not empty, but they did not characterize it further.

Theorem 3.2. Assume that (2.1), the imitation of the most successful action dynamics, (2.5), and the strict rule (2.7) hold.

(i) If $a+b>(c+3 d) / 2$ then $S_{0}=\{\boldsymbol{C}, \boldsymbol{D}\}, S_{*}=\{\boldsymbol{D}\}$, and $\mathrm{E}_{\varepsilon}(T) \approx \varepsilon^{-1}$.

(ii) If $a+b \leq(c+3 d) / 2$ and $(3 a+b) / 2<c+d$, then $S_{0}=\{\boldsymbol{C}, \boldsymbol{D}\} \cup M$, where the mixed stationary states in $M$ are as shown in (3.2) with all $d_{i} \in\{1,2,3\}$ and, besides $c_{i} \geq 3$,

$$
\begin{aligned}
c_{i} \geq 5 \quad \text { if }\left(d_{i}, d_{i+1}\right) & =(1,1) \\
\text { and } \quad c_{i} \geq 4 \quad \text { if }\left(d_{i}, d_{i+1}\right) & =(1,2) \text { or }(2,1) .
\end{aligned}
$$

Moreover, as $\varepsilon \downarrow 0$,

$$
\begin{gathered}
S_{*}=\{\boldsymbol{D}\} \quad \text { and } \quad \mathrm{E}_{\varepsilon}(T) \approx \varepsilon^{-1} \text { for } n=5, \\
S_{*}=\{\boldsymbol{D}\} \quad \text { and } \mathrm{E}_{\varepsilon}(T) \approx \varepsilon^{-\lceil n / 10\rceil} \quad \text { for } 6 \leq n \leq 20, \\
S_{*}=S_{0} \text { and } \mathrm{E}_{\varepsilon}(T) \approx \varepsilon^{0} \quad \text { for } 21 \leq n<30 \text { but } n \neq 25, \\
S_{*}=S_{0} \backslash M_{2,3} \quad \text { and } \mathrm{E}_{\varepsilon}(T) \approx \varepsilon^{-1} \text { for } n=25 \text { or } 30, \\
S_{*}=\left(S_{0} \backslash M_{2,3}\right) \backslash\{\boldsymbol{D}\} \quad \text { and } \mathrm{E}_{\varepsilon}(T) \approx \varepsilon^{-3} \text { for } n \geq 31,
\end{gathered}
$$

where $\lceil x\rceil$ is the least integer greater than or equal to $x$. 
(iii) If $a+b \leq(c+3 d) / 2$ and $(3 a+b) / 2 \geq c+d$, then $S_{0}=\{\boldsymbol{C}, \boldsymbol{D}\} \cup M_{\geq 2, \geq 3}$. Moreover, $S_{*}=\{\boldsymbol{D}\}$ and $\mathrm{E}_{\varepsilon}(T) \approx \varepsilon^{-1}$ as $\varepsilon \downarrow 0$.

Note that $M_{2,3} \neq \varnothing$ if and only if $5 \mid n$, where $5 \mid n$ means that $n$ is a multiple of 5 .

In view of Theorem 3.1, it is no surprise to expect $\boldsymbol{D}$ to be the unique long-run equilibrium indicated in Theorem 3.2(i) and (iii). Yet, Theorem 3.2(ii) shows that, when $a+b \leq(c+3 d) / 2$ and $(3 a+b) / 2<c+d, S_{*}$ varies as the population size $n$ grows from $\{\boldsymbol{D}\}=S_{*}$ for $5 \leq n \leq 20$ to $\{\boldsymbol{D}, \boldsymbol{C}\} \subset S_{*}$ for $21 \leq n \leq 30$ and finally to $S_{*}=\left(S_{0} \backslash M_{2,3}\right) \backslash\{\boldsymbol{D}\}$ for $n \geq 31$. In particular, full cooperation, $\boldsymbol{C}$, instead of full defection, $\boldsymbol{D}$, could emerge as a long-run equilibrium under the imitation of the most successful action dynamics when the number of players is over 30 .

In the following we demonstrate why full cooperation could emerge as a long-run equilibrium under the imitation of the most successful action dynamics, but not under the imitation of the most successful player dynamics. Consider that a $D$-string having length greater than or equal to 3 confronts a $C$-string having length greater than or equal to 2 . Then players' payoffs under the imitation of the most successful player dynamics are as follows:

$$
\begin{array}{cccccl}
\cdots D & D & D & C & C \cdots & \text { (state), } \\
& 2 a & (a+b) & (c+d) & & \text { (payoff). }
\end{array}
$$

Since $a+b>\max \{c+d, 2 a\}$, the $D$-player on the boundary will win and hold. However, under the imitation of the most successful action dynamics, the average payoffs of strategies $D$ and $C$ are

$$
a^{D}=\frac{2 a+(a+b)}{2}=\frac{3 a+b}{2} \text { and } a^{C}=c+d,
$$

respectively. With $(3 a+b) / 2<c+d$, the $D$-player on the boundary cannot be sustained; hence, the $C$-player will win and expand. This is because the force deviating from cooperation, i.e. payoff $(b)$, is weakened in the process of averaging neighbors' payoffs. Accordingly, the states with a single $D$,

$$
\cdots C C C \dot{D} C C C \cdots,
$$

could be mixed stationary states under the imitation of the most successful action dynamics, but not under the imitation of the most successful player dynamics. By these mixed stationary states, fewer mutants are required to transfer states in $M$ to $C$ under the imitation of the most successful action dynamics than under the imitation of the most successful player dynamics. Hence, $\boldsymbol{C}$ could be the long-run equilibrium under the imitation of the most successful action dynamics, but not under the imitation of the most successful player dynamics.

Next, our results are compared with those of Eshel et al. (1998). Let $d-c=b-a=1$, $a=0$, and define $\alpha=2(a-c) /(d-c)=-2 c$. A simple calculation shows that the payoff function $z_{i}(s)$ in (2.2) becomes

$$
z_{i}(s)= \begin{cases}n_{i}^{C}(s) & \text { if } s_{i}=D, \text { i.e. player } i \text { chooses strategy } D, \\ n_{i}^{C}(s)-\alpha & \text { if } s_{i}=C, \text { i.e. player } i \text { chooses strategy } C .\end{cases}
$$

With this payoff function, the imitation of the most successful action dynamics has been studied in Eshel et al. (1998), where players choosing strategies $C$ and $D$ are called altruists and egoists, respectively. Note that (2.1) implies that $-1<c<0$ and, thus, $0<\alpha<2$. It is easy to check that Theorem 3.2(i), (ii), and (iii) correspond to the cases $\alpha>0.5,0<\alpha<0.5$, and $\alpha=0.5$, respectively. For $n \geq 31$ and $0<\alpha<0.5$, Eshel et al. (1998) showed that

$$
S_{*} \subseteq S_{0} \subseteq\left\{s \in S:\left|\left\{i \in N: s_{i}=C\right\}\right| \geq 0.6 n\right\} .
$$


That is, only those states with the cooperator proportion no less than 0.6 could possibly be LRE. In particular, $\boldsymbol{D} \notin S_{*}$. However, they could not decide whether $\boldsymbol{C} \in S_{*}$ or $M_{2,3} \subseteq S_{*}$, as they lacked complete information about $S_{0}$ and $S_{*}$. Note that $M_{2,3} \subset\left\{s \in S:\left|\left\{i \in N: s_{i}=C\right\}\right| \geq\right.$ $0.6 n$ \}. In contrast, Theorem 3.2 describes $S_{*}$ and $S_{0}$ explicitly for all PD game payoffs with population size no less than 5 .

In comparison with Theorem 3.1 for the imitation of the most successful player dynamics, Theorem 3.2 shows that the convergence rate to $S_{*}$ under the imitation of the most successful action dynamics depends on the game payoff structure and population size. Robson and VegaRedondo (1996) showed that $\boldsymbol{D}$ is the unique long-run equilibrium, and its convergence rate has order $\varepsilon^{-1}$ under the global interaction setup. Theorem 3.2(i) and (iii) show the same conclusion under the local interaction setup. However, Theorem 3.2(ii) indicates that, for $11 \leq n \leq 20$, the convergence rate to $S_{*}=\{\boldsymbol{D}\}$ has order $\varepsilon^{-2}$, which is slower than that under global interaction. This outcome is contrary to that of Ellison (1993), who showed that the convergence rate to $S_{*}$ under local interaction is faster than that under global interaction when the best-reply dynamics were adopted. Moreover, Theorem 3.2(ii) shows that the convergence rate to $S_{*}$ has order $\varepsilon^{-3}$ for $n \geq 31$.

Finally, we remark that the method developed in this paper can be applied to other $2 \times 2$ symmetric games like the coordination games and other updating schemes, such as players matching their neighbors randomly for $1 \leq v \leq \infty$ rounds. These results are reported in Chen et al. (2007a), (2007b) and Chow and Wu (2009). Note that, by the strong law of large numbers, the case in which $v=\infty$ is equivalent to players matching once with each of their two neighbors and is investigated in this paper. Theorem 3.2 is not so satisfactory, as full cooperation must coexist with other LRE. It is interesting to know whether full cooperation could prevail as the unique long-run equilibrium for other spatial structures like the two-dimensional lattices.

\section{The method of Freidlin and Wentzell}

Some terminologies are needed in order to describe the invariant distribution $\mu_{\varepsilon}$ in (2.13). Let $W$ be a subset of $S$. A graph $g$ consisting of arrows $\boldsymbol{u} \rightarrow \boldsymbol{v}$, where $\boldsymbol{u} \in S \backslash W$ and $\boldsymbol{v} \in S$, is called a $W$-graph if it satisfies the following conditions: (i) every state in $S \backslash W$ is the beginning of exactly one arrow; (ii) there exists a sequence of arrows leading from any state in $S \backslash W$ to $W$. Or, equivalently, there are no cycles in the graph $g$.

Denote by $G(W)$ the set of all $W$-graphs. For any state $s \in S$, define

$$
\alpha_{s}=\sum_{g \in G(\{s\})} \prod_{(\boldsymbol{u} \rightarrow \boldsymbol{v}) \in g} Q_{\varepsilon}(\boldsymbol{u}, \boldsymbol{v}) .
$$

It is shown in Freidlin and Wentzell (1984, p. 177) that $\left(\alpha_{s}: s \in S\right)=\left(\alpha_{s}: s \in S\right) Q_{\varepsilon}$. Since $\mu_{\varepsilon}$ is the unique probability distribution satisfying (2.13), it follows that

$$
\mu_{\varepsilon}(s)=\frac{\alpha_{s}}{\sum_{t \in S} \alpha_{t}} \quad \text { for each } s \in S
$$

Equation (4.2) holds for any time-homogeneous, irreducible, and aperiodic finite-state Markov chain. In general, it is not useful, as computing $\alpha_{s}$ from (4.1) is difficult. However, from (2.12) we have, for any $\boldsymbol{s}, \boldsymbol{u} \in S$,

$$
Q_{\varepsilon}(\boldsymbol{s}, \boldsymbol{u})=\varepsilon^{U(\boldsymbol{s}, \boldsymbol{u})}(1+O(\varepsilon)),
$$


where $U(\boldsymbol{s}, \boldsymbol{u})=d(\boldsymbol{r}(\boldsymbol{s}), \boldsymbol{u})=\left|\left\{i \in N: u_{i} \neq r_{i}(\boldsymbol{s})\right\}\right|$ and $\boldsymbol{r}(\boldsymbol{s})$ is the rational choice uniquely determined under the strict rule (2.7). Hence,

$$
\alpha_{s}=\sum_{g \in G(\{s\})} \varepsilon^{v(g)}(1+O(\varepsilon)) \approx \varepsilon^{v(\{s\})} \text { for small } \varepsilon,
$$

where $v(g)=\sum_{(\boldsymbol{u} \rightarrow \boldsymbol{v}) \in g} U(\boldsymbol{u}, \boldsymbol{v})$ and $v(\{\boldsymbol{s}\})=\min _{g \in G(\{\boldsymbol{s}\})} v(g)$ are constants independent of $\varepsilon$. Define

$$
v_{1}=\min _{\boldsymbol{s} \in S} v(\{s\})
$$

By (4.2) and (4.4), $\mu_{*}=\lim _{\varepsilon \rightarrow 0} \mu_{\varepsilon}$ does exist and the following holds.

Theorem 4.1. The support $S_{*}$ of $\mu_{*}$ defined in (2.14) is given by

$$
S_{*}=\left\{\boldsymbol{s} \in S \mid v(\{\boldsymbol{s}\})=v_{1}\right\} \quad \text { and } \quad \mu_{\varepsilon}(\boldsymbol{u}) \approx \varepsilon^{v(\{\boldsymbol{u}\})-v_{1}} \quad \text { for any } \boldsymbol{u} \in S .
$$

In order to find the order estimate of $\mathrm{E}_{\varepsilon}(T)$ defined in (2.15), we need to generalize (4.5). For $k \geq 1$, define

$$
v_{k}=\min _{|W|=k} v(W), \quad \text { where } \quad v(W)=\min _{g \in G(W)} v(g) .
$$

Let $W_{k}$ be any solution to $v_{k}$ above. Note that $W_{1} \subseteq S_{*}$. Since $W_{k}=S$ when $k=|S|, W_{k} \subseteq S_{*}$ cannot always be valid unless $S_{*}=S$. As will be shown in (5.9), below, this exceptional case does not fit our models discussed in Section 2.

Theorem 4.2. (Chiang and Chow (2007).) Let $T$ be as given in (2.15). Then

$$
\mathrm{E}_{\varepsilon}(T) \approx \varepsilon^{-\delta} \quad \text { as } \varepsilon \downarrow 0,
$$

where $\delta=v_{k_{0}-1}-v_{k_{0}}$ and $k_{0}=\min \left\{k \geq 2:\right.$ there exists $W \subseteq S$ with $|W|=k, v(W)=v_{k}$, and $\left.W \nsubseteq S_{*}\right\}$.

The constant $\delta$ above means 'escape energy' in simulated annealing, which is a probabilistic algorithm for finding the global minima of combinatorial optimization problems. See Kirkpatrick et al. (1983) and Geman and Geman (1984) for an introduction on this topic. With this amount of energy, any state outside $S_{*}$, the so-called 'global minima' set, could reach $S_{*}$. See Chiang and Chow (1989) for how to obtain $S_{*}$ by the cycle method.

Regarding $U(\boldsymbol{u}, \boldsymbol{v})$ as the cost of going from $\boldsymbol{u}$ to $\boldsymbol{v}$, then $v(\{\boldsymbol{s}\})$ means the minimum cost of all spanning trees with root at $s$. By (4.6), the set $S_{*}$ consists of those states in $S$ which attain the minimum cost $v_{1}$ when treated as a root. Similarly, any solution $W_{k}$ to (4.7) represents an optimal choice for attaining $v_{k}$, the minimum cost of all spanning forests with $k$ roots on $S$. The quantity $\left(v_{k}-v_{k+1}\right)$ is then the cost saved from having $k$ roots to having $k+1$ roots in constructing optimal spanning forests on $S$.

\section{Proofs of Theorems 3.1 and 3.2}

In view of Theorems 4.1 and 4.2, the method of Freidlin and Wentzell (1984) can be used to find the support $S_{*}$ and the order estimate of $\mathrm{E}_{\varepsilon}(T)$. The procedure is as follows.

Since $S_{*} \subseteq S_{0}$ by (2.16), the first step is to find $S_{0}$, which is the set of all stationary states under $Q_{0}$. Let $s \in S \backslash S_{0}$ be any transient state. By definition, there exist $s_{0}=s, s_{1}, \ldots, s_{j}$ such 
that $s_{j} \in S_{0}$ and $Q_{0}\left(\boldsymbol{s}_{k}, \boldsymbol{s}_{k+1}\right)>0$ for $0 \leq k<j$. By (2.8) and (4.3), $\sum_{k=0}^{k=j-1} U\left(\boldsymbol{s}_{k}, \boldsymbol{s}_{k+1}\right)=0$. The converse does not hold. Otherwise, $s \in S_{0}$. In summary,

$s$ is transient if and only if there exists a zero-cost path from $s$ to $S_{0}$,

but the converse does not hold.

In the case in which $M \neq \varnothing$, any $s \in M$ has the form shown in (3.2). We need to find conditions on the $d_{j}$ and $c_{j}$ in (3.2) in order that $s \in M$.

The next step is to compute $v(\{\boldsymbol{s}\})$, the minimum-cost spanning tree rooted at $s \in S$, and solutions $W_{k}$ to (4.7). Then $S_{*}$ and $\mathrm{E}\left(T_{\varepsilon}\right)$ can be obtained via (4.6)-(4.8). It suffices to consider $s \in S_{0}$, as (5.1) shows that there is no advantage in choosing any transient $\boldsymbol{u}$ as a root. Hence, $\boldsymbol{u} \notin S_{*}$. By the same argument, $\boldsymbol{u} \notin W$ for any solution $v(W)=v_{k}$ in (4.7) unless no stationary state in $S_{0}$ remains available. In particular,

$$
\text { if } S_{*}=S_{0} \text { then } \mathrm{E}_{\varepsilon}(T) \approx \varepsilon^{0} \text { as } k_{0}=\left|S_{0}\right|+1 \text { and } \delta=0 \text { in (4.8). }
$$

We can decompose $S_{0}$ into disjoint closed connected components. By (2.9), $\{\boldsymbol{C}\}$ and $\{\boldsymbol{D}\}$ are two examples. Let $F$ be any closed connected component of $S_{0}$ under $Q_{0}$. Then

$$
U(\boldsymbol{s}, \boldsymbol{u}) \geq 1 \quad \text { for any } \boldsymbol{s} \in F \text { and } \boldsymbol{u} \notin F,
$$

as $Q_{0}(\boldsymbol{s}, \boldsymbol{u})=0$ for $s \in F$ and $\boldsymbol{u} \notin F$. Hence, it pays to reach out from $F$. Define

$$
V(s)=\text { the minimum cost of paths from } s \in F \text { to some } \boldsymbol{u} \text { in } S_{0} \backslash F .
$$

Being a closed connected component under $Q_{0}$, any two states in $F$ can be connected by a path in $F$ with zero total cost. Hence, when constructing a minimum-cost spanning forest, all states in $F$ should first converge to a certain state of $F$ and then reach out from there in case the roots of the forest lie outside $F$. Only the last reach-out move will cost some price by (5.3). Therefore,

$$
V(s) \geq 1 \text { is a constant on any closed connected component } F \subseteq S_{0} .
$$

In fact, the constant is $\min \left\{U(\boldsymbol{w}, \boldsymbol{u}): \boldsymbol{w} \in F\right.$ and there exists a zero-cost path from $\boldsymbol{u}$ to $\left.S_{0} \backslash F\right\}$. We will show later that $V(s)=1$ for any $s \in M$. More importantly,

all states in $M$ can reach $\boldsymbol{D}$ at minimum cost 1 per connected component.

This is the key ingredient that makes the method of minimum-cost spanning forests efficient for determining $S_{*}$ and $\mathrm{E}\left(T_{\varepsilon}\right)$ via (4.6)-(4.8).

For convenience, we introduce the following notation:

- $s \stackrel{k}{\rightarrow} \boldsymbol{u}$ means that $U(\boldsymbol{s}, \boldsymbol{u})=k$;

- $\boldsymbol{s} \stackrel{k}{\leftrightarrow} \boldsymbol{u}$ means that $U(\boldsymbol{s}, \boldsymbol{u})=k$ and $U(\boldsymbol{u}, \boldsymbol{s})=k$.

Also, note that $r_{i}(s)$ depends only on the strategies $\left(s_{i-2}, s_{i-1}, s_{i}, s_{i+1}, s_{i+2}\right)$ adopted by five consecutive players from $i-2$ to $i+2$ and are, in fact, independent of the time $t$ and the label of player $i$. For brevity, we define

$$
r\left(s_{i-2}, s_{i-1}, s_{i}, s_{i+1}, s_{i+2}\right):=r_{i}(s) .
$$


In the following, ' $*$ ' denotes $C$ or $D$ independently. Tables 1 and 2 in Appendix A imply that

$$
r(*, C, D, C, *)=D \quad \text { and } \quad r(*, D, C, D, *)=D,
$$

which shows the strength of $D$ against $C$. By (2.6) we also have

$$
r(*, D, D, D, *)=D \quad \text { and } \quad r(*, C, C, C, *)=C .
$$

Since $c=\min \{a, b, c, d\}$ by (2.1), we have $\boldsymbol{r}(\boldsymbol{s})=\boldsymbol{D}$ and then $U(\boldsymbol{s}, \boldsymbol{D})=0$ for any $\boldsymbol{s} \in S$ with $n^{C}(s):=\left|\left\{j \in N: s_{j}=C\right\}\right|=1$. Hence, by (2.9), (2.16), and (5.1),

$$
S_{*} \subseteq S_{0} \subseteq S \backslash\left\{s: n^{C}(s)=1\right\} \neq S .
$$

This verifies the remark after (4.7). Now we are ready to prove Theorems 3.1 and 3.2.

\subsection{Proof of Theorem 3.1 for the imitation of the most successful player dynamics}

Part (i): $a+b>2 d$. By symmetry and (2.1), it follows from Table 4 in Appendix A that

$$
r(*, D, C, C, *)=r(*, C, C, D, *)=D .
$$

Note that $a+b>c+d$ and $b=\max \{a, b, c, d\}$ by (2.1). Similarly, Table 3 in Appendix A implies that

$$
r(*, C, D, D, *)=r(*, D, D, C, *)=D .
$$

Equations (5.7)-(5.8) and (5.10)-(5.11) show that any $D$-string in a mixed state $s$ will grow under $Q_{0}$ by absorbing its neighboring $C$ s. Hence,

$$
\text { any } \boldsymbol{s} \in S \backslash\{\boldsymbol{C}, \boldsymbol{D}\} \text { will reach state } \boldsymbol{D} \text { at zero total cost under } Q_{0} \text {. }
$$

By (2.11) and (5.1), $S_{0}=\{\boldsymbol{C}, \boldsymbol{D}\}$. We claim that

$$
v(\{\boldsymbol{D}\})=1 \quad \text { and } \quad v(\{\boldsymbol{C}\})=n .
$$

The former follows from (5.12) since $U(\boldsymbol{C}, \boldsymbol{s})=1$ for any state $s$ with $n^{C}(\boldsymbol{s})=n-1$. Equation (5.12) also implies the latter, as the minimum-cost path from $\boldsymbol{D}$ to $\boldsymbol{C}$ is to jump directly to $\boldsymbol{C}$ at cost $U(\boldsymbol{D}, \boldsymbol{C})=n$ due to $r(\boldsymbol{D})=\boldsymbol{D}$.

Using (4.6) and (5.13), we easily obtain the desired conclusions:

$$
v_{1}=1, \quad S_{*}=\{\boldsymbol{D}\}, \mu_{\varepsilon}(\boldsymbol{C}) \approx \varepsilon^{n-1}, \quad \text { and } \quad \mathrm{E}_{\varepsilon}(T) \approx \varepsilon^{-1},
$$

as $v(\{\boldsymbol{D}, \boldsymbol{C}\})=v_{2}=0$ in (4.7) and then $k_{0}=2$ and $\delta=v_{1}-v_{2}=1$ in (4.8).

Part (ii): $a+b \leq 2 d$. While (5.11) still holds from Table 3, Table 4 implies that

$$
r\left(s_{2}, C, C, D, s_{1}\right)=r\left(s_{1}, D, C, C, s_{2}\right)=C \quad \text { if and only if } \quad s_{1}=D \text { and } s_{2}=C,
$$

which is different from (5.10) in part (i). By (5.11) and (5.15),

any $D$-string with length greater than or equal to 2 in a state can hold under $Q_{0}$,

while a $C$-string in a state can hold only when it has length greater than or equal to 3 and is surrounded by $D$-strings with length greater than or equal to 2 . In particular, $M_{\geq 2, \geq 3} \subseteq M$. By (5.7), (5.11), and (5.15),

any $C$-string with length less than or equal to 2 is eliminated at the next period under $Q_{0}$, 
while a $D$-string with length greater than or equal to 3 will then form at this position in the next period. By (5.16), this $C$-string can never reappear at its original position. Hence, any $C$-string in a state $s \in M$ must have length greater than or equal to 3. Moreover, by (5.7),

no singleton $D$-string in a state could be eliminated under $Q_{0}$.

In fact, it will grow to a length greater than or equal to 3 under $Q_{0}$ and then can never be diminished by (5.16). Hence, any $D$-string in $s \in M$ has length greater than or equal to 2 . Putting together, $M \subseteq M_{\geq 2, \geq 3}$ and, thus, $M=M_{\geq 2, \geq 3}$. It is also clear from the above arguments that $\boldsymbol{r}(\boldsymbol{s})=\boldsymbol{s}$ for any $\boldsymbol{s} \in M$. Together with (2.9), each state in $S_{0}$ is an absorbing state under $Q_{0}$.

Next we study how states in $S_{0}=M \cup\{\boldsymbol{C}, \boldsymbol{D}\}$ communicate with each other in order to find $v(\{s\})$ for $s \in S_{0}$. Decompose $M=M_{\geq 2, \geq 3}$ as follows:

$$
M=\bigcup_{1}^{L} M_{k}, \quad \text { where } \quad M_{k}:=\{s \in M: s \text { has } k \text { disjoint } D \text {-strings in (3.2) }\} .
$$

Here $L=\lfloor n / 5\rfloor$, where $\lfloor x\rfloor$ denotes the greatest integer less than or equal to $x$. In particular,

if $5 \mid n$ then $M_{L}=M_{2,3}$; that is, all $d_{i}=2$ and $c_{i}=3$ in (3.2) for $s \in M_{L}$.

We verify (5.6) in the following three steps. Remember that $V(s) \geq 1$ for $s \in S_{0}$ by (5.5).

Step 1. Let $s \in M_{k}$ be as shown in (3.2). If $d_{i} \geq 3$ then

$$
\begin{aligned}
& \cdots \underbrace{C \cdots C}_{c_{i-1}} \underbrace{\stackrel{\circ}{D} \cdots D}_{d_{i}-1} \underbrace{\stackrel{\bullet}{C} \cdots C}_{c_{i}+1} \cdots \stackrel{1}{\leftrightarrow} \cdots \underbrace{C \cdots C}_{c_{i-1}} \underbrace{\stackrel{\circ}{D} \cdots \dot{D}}_{d_{i}} \underbrace{C \cdots C}_{c_{i}} \cdots \\
& \stackrel{1}{\leftrightarrow} \cdots \underbrace{C \cdots C \stackrel{\circ}{C}}_{c_{i-1}+1} \underbrace{D \cdots \dot{D}}_{d_{i}-1} \underbrace{C \cdots C}_{c_{i}} \cdots .
\end{aligned}
$$

In words, one strategy $C$, marked by a ' $\bullet$ ', of the left state is mutated to a $D$ strategy, also marked by a ' $\bullet$ ', and, thus, reaches the middle state $s$. The strategy where mutation occurs between $s$ and the last state is marked by a 'o'. All three states above lie in $M_{k}$ due to $d_{i} \geq 3$. Hereafter, we use a ' $\bullet$ ' to mark a certain player (or players) whose strategy might change in the process shown in the diagram. When necessary, ' $\circ$ ' and ' $*$ ' over a strategy are used to indicate where mutation occurs.

Repeating the same procedures, any $D$-string in $s$ can be trimmed to its minimum length 2 at the minimum cost 1 for each move while all the encountered states remain in $M_{k}$. Note that the path is reversible at the same cost.

Step 2. Assume that $5 \nmid n$ or $1 \leq k<L$. For any $\boldsymbol{s}, \boldsymbol{u} \in M_{k}$, there is a path in $M_{k}$ connecting $\boldsymbol{s}$ and $\boldsymbol{u}$ as follows:

$$
\boldsymbol{s} \stackrel{1}{\leftrightarrow} s_{1} \stackrel{1}{\leftrightarrow} s_{2} \stackrel{1}{\leftrightarrow} \cdots \stackrel{1}{\leftrightarrow} s_{j-1} \stackrel{1}{\leftrightarrow} u \quad \text { and, thus, } \quad V(s)=1 \quad \text { by (5.4). }
$$

By step 1, we may assume without loss of generality that all $D$-strings in $\boldsymbol{s}$ and $\boldsymbol{u}$ have length 2. Let $s \in M_{k}$ be as shown in (3.2). If $c_{i} \geq 4$ then

$$
\begin{array}{r}
\cdots \underbrace{C \cdots C}_{c_{i-1}} \underbrace{D \dot{D}}_{2} \underbrace{\stackrel{\circ}{C} C \cdots C}_{c_{i}} \cdots \stackrel{\leftrightarrow}{\leftrightarrow} \cdots \underbrace{C \cdots C}_{c_{i-1}} \underbrace{\stackrel{\bullet}{D} \dot{\circ} D}_{3} \underbrace{C \cdots C}_{c_{i}-1} \cdots \\
\stackrel{1}{\leftrightarrow} \cdots \underbrace{C \cdots C}_{c_{i-1}+1} \stackrel{\stackrel{*}{C}}{\stackrel{\bullet}{D} D} \underbrace{C \cdots C}_{2} \cdots
\end{array}
$$


and all three states above lie in $M_{k}$. This shows that any extra $C$ in a $C$-string of $s$ can be shifted either to its left or to its right $C$-string at cost 1 , which is the minimum by (5.5). Moreover, the path is reversible at the same cost. Repeating the same procedures, all extra $C s$ in $s$ can be arbitrarily distributed among its $C$-strings at the minimum cost 1 for each move while always staying in $M_{k}$. The existence of an extra $C$ in $s$ is crucial for such shiftings in (5.22). Since now all $d_{i}=2$ in (3.2) for $s,(5.20)$ implies that there is at least $\min (n-5 L, 5) \geq 1$ extra $C$ s in $s$ due to $5 \nmid n$ or $k<L$. This verifies (5.21). Note that $U(\boldsymbol{s}, \boldsymbol{u}) \geq 2 L$ for two different $\boldsymbol{s}, \boldsymbol{u}$ in $M_{L}$ in the case in which $5 \mid n$.

Step 3. Consider $s \in M_{k}$ with $1 \leq k \leq L$. Using (5.20) in the case in which $5 \mid n$ and $k=L$, and using step 2 otherwise, we may assume without loss of generality that all $c_{i}=3$ in (3.2) for $s$. By (5.7) and (5.11),

$$
\begin{gathered}
\cdots \underbrace{D \cdots D}_{d_{i}} \underbrace{C \dot{C} C}_{3} \underbrace{D \cdots D}_{d_{i+1}} \cdots \stackrel{1}{\rightarrow} \cdots \underbrace{D \cdots D}_{d_{i}} \underbrace{C \dot{D} C}_{3} \underbrace{D \cdots D}_{d_{i+1}} \cdots \\
\stackrel{0}{\rightarrow} \cdots \underbrace{D \cdots D}_{d_{i}+3+d_{i+1}} \cdots \in M_{k-1} .
\end{gathered}
$$

Repeating the same procedure $k-1$ times, we conclude that all states in $M$ can reach $\boldsymbol{D}$ at the minimum cost 1 per state. This verifies (5.6).

It remains to study how $\boldsymbol{C}$ and $\boldsymbol{D}$ reach out. By (5.18), the following diagram is optimal for $n=5$ :

$$
M_{1} \ni \boldsymbol{u}:=\stackrel{\circ}{D} \stackrel{\circ}{D} C C C \stackrel{2}{\rightarrow} \boldsymbol{C} \stackrel{1}{\rightarrow} C C \stackrel{\bullet}{D} C C \stackrel{0}{\rightarrow} C D \stackrel{\bullet}{D} D C \stackrel{0}{\rightarrow} \boldsymbol{D} .
$$

Similarly, we have the following optimal path for $n \geq 6$ :

$$
M_{1} \ni \boldsymbol{u}:=\stackrel{\circ}{D} \stackrel{\circ}{D} \underbrace{C \cdots C}_{n-2} \stackrel{2}{\rightarrow} \boldsymbol{C} \stackrel{1}{\rightarrow} C \stackrel{\dot{D}}{\underbrace{C \cdots C}_{n-2} \stackrel{0}{\rightarrow} D} \dot{D} D \underbrace{C \cdots C}_{n-3 \geq 3} \in M_{1} .
$$

Hence, $V(\boldsymbol{C})=1$ for $n \geq 5$. On the other hand, (5.17) implies that the following path is optimal for $\boldsymbol{D}$ to reach out:

$$
\boldsymbol{D} \stackrel{3}{\rightarrow} \underbrace{D \cdots D}_{n-3 \geq 2} \stackrel{\circ}{\mathrm{C}} \stackrel{\circ}{\mathrm{C}} \stackrel{\circ}{\mathrm{C}} \in M_{1} .
$$

It follows, from (5.5)-(5.6) and (5.23)-(5.25), that

$v(\{\boldsymbol{C}\})=|M|+4, \quad v(\{\boldsymbol{D}\})=|M|+1, \quad$ and $\quad v(\{\boldsymbol{s}\}) \geq|M|+3 \quad$ for $\boldsymbol{s} \in M=M_{\geq 2, \geq 3}$.

We obtain the first equation above by requiring that all states in $M \cup\{\boldsymbol{D}\}$ converge to $\boldsymbol{u}$ in (5.23) or (5.24), and then from $\boldsymbol{u}$ to $\boldsymbol{C}$ at cost 2. Hence, $v_{1}=v(\{\boldsymbol{D}\})=|M|+1, S_{*}=\{\boldsymbol{D}\}$, and $\mu_{\varepsilon}(\boldsymbol{C}) \approx \varepsilon^{3}$ by (4.6). Then $k_{0}=2$ in (4.8) as $v_{2}=v(\{\boldsymbol{s}, \boldsymbol{D}\})=|M|$ for any $\boldsymbol{s} \in M_{1} \cup\{\boldsymbol{C}\}$. Finally, $\delta=v_{1}-v_{2}=1$, as desired.

\subsection{Proof of Theorem 3.2 for the imitation of the most successful action dynamics}

Remember that (5.7) and (5.8) remain valid. Tables 3 and 4 in Appendix A imply that the rational choice $\boldsymbol{r}(\boldsymbol{s})$ defined in (2.7) depends on the relative magnitudes of $a+b,(3 a+b) / 2, c+d$, and $(c+3 d) / 2$. Note that, under $(2.1)$,

$$
2 b>\frac{c+3 d}{2}>c+d, \quad a+b>\frac{3 a+b}{2}>2 c, \quad \text { and } \quad a+b>c+d>2 c .
$$


It remains to compare $(3 a+b) / 2$ with $c+d$ and $a+b$ with $(c+3 d) / 2$. This leads to the following classifications.

Part (i): $a+b>(c+3 d) / 2$. By the results in Table 4 and (5.26), (5.10) holds. In the subcase $(3 a+b) / 2 \geq c+d$, the results in Table 3 and (5.26) imply that (5.11) holds. As in Theorem 3.1(i), any $D$-string in a mixed state $s$ will grow by absorbing its neighboring $C$ s. As a consequence, (5.12) holds.

For the remaining subcase $(3 a+b) / 2<c+d$, we now show that (5.12) still holds. Instead of (5.11), from the results in Table 3 and (5.26), we obtain

$$
r\left(s_{2}, C, D, D, s_{1}\right)=r\left(s_{1}, D, D, C, s_{2}\right)=C \quad \text { if and only if } s_{1}=D \text { and } s_{2}=C .
$$

By (5.7), (5.10), and (5.27), any $D$-string with length less than or equal to 2 in a mixed state $s$ will absorb its two neighboring $C$ s and, thus, form a $D$-string of length greater than or equal to 3 in the next period under $Q_{0}$. Similarly, we have, for $d \geq 3$,

$$
\begin{aligned}
& \cdots D \dot{C} \underbrace{D \cdots D}_{d \geq 3} \dot{C} D \cdots \stackrel{0}{\rightarrow} \cdots \underbrace{D \dot{D} D \cdots D \dot{D} D}_{d+4} \cdots, \\
& \cdots D C \underbrace{\dot{D} \cdots D \dot{D}}_{d \geq 3} C C \cdots \stackrel{0}{\rightarrow} \cdots \underbrace{D D \dot{D} \cdots D}_{d+1} \dot{C} D * \cdots .
\end{aligned}
$$

Here and hereafter, any $*=C$ or $D$ and is undecidable at present. Moreover,

$$
\cdots C C \underbrace{\stackrel{\dot{D} D \cdots D}{D} \boldsymbol{D}}_{d \geq 3} C C \cdots \stackrel{0}{\rightarrow} \cdots D \underbrace{\stackrel{\bullet}{C} D \cdots D \dot{C}}_{d} D \cdots \stackrel{0}{\rightarrow} \cdots * \underbrace{D \dot{D} D \cdots D \dot{D} D}_{d+2} * \cdots .
$$

It follows that any $D$-string with length greater than or equal to 3 in a mixed state $s$ will grow in the next period or in one more period later under $Q_{0}$. By repeating the same procedures, this verifies (5.12) for the subcase $(3 a+b) / 2<c+d$.

By (2.11) and (5.1), $S_{0}=\{\boldsymbol{C}, \boldsymbol{D}\}$. As in Theorem 3.1(i), (5.13) holds and then (5.14) follows.

Part (ii): $a+b \leq(c+3 d) / 2$ and $(3 a+b) / 2<c+d$. By the results in Table 3 we still have (5.27), which means that any $D$-string of length greater than or equal to 3 will diminish at the next period under $Q_{0}$ if surrounded by nonsingleton $C$-strings. Because of this property, the structure of $S_{0}$ is complicated, as indicated in (3.3). The results in Table 4 and (5.26) imply (5.15). In particular, $M_{2, \geq 3} \subseteq M$ by (5.15) and (5.27). The following statements, (S1)-(S5), verify that $M$ is as claimed in (3.3).

(S1) By (5.1), $u \in M$ if $s \in M$ and $s \stackrel{0}{\rightarrow} u$.

(S2) Any $C$-string in a state $s \in M$ must have length greater than or equal to 3 .

Statement (S2) holds because (5.7), (5.15), and (5.27) imply that any $C$-string with length less than or equal to 2 in a state $s$ will be eliminated at the next period, as shown in

$$
\cdots D \dot{C} D \cdots \stackrel{0}{\rightarrow} \cdots D \dot{D} D \cdots \quad \text { and } \quad \cdots C * D \dot{C} \dot{C} D * C \cdots \stackrel{0}{\rightarrow} \cdots * * D \dot{D} \dot{D} D * * \cdots
$$

and (5.29), below. Here each ' $*$ ' can be $C$ or $D$. Though some new $C$ s could be generated, which can happen by (5.27) if the $C$-string is of length 2 and is confronted with a $D$-string with 
length greater than or equal to 3 , they are always isolated and will disappear in the next period:

$$
\cdots D D \dot{D} \underbrace{C C}_{2} \dot{D} D D \cdots \stackrel{0}{\rightarrow} \cdots * D \dot{C} \underbrace{D D}_{2} \dot{C} D * \cdots \stackrel{0}{\rightarrow} \cdots * D \dot{D} \underbrace{D D}_{2} \dot{D} D * \cdots .
$$

(S3) Any $D$-string in a state $s \in M$ must have length 1,2 , or 3 .

By (S1) and (S2), we may assume that all $C$-strings encountered hereafter have length greater than or equal to 3. By (5.27), any $D$-string with length $d \geq 3$ in $s \in M$ will shrink by 2 per period under $Q_{0}$. So its length will drop to 1 or 2 at some stage. By (5.15) and (5.27), a $D$-string of length 2 can hold under $Q_{0}$, while a singleton $D$-string will grow by (5.7) and (5.15) to length 3 and then back to length 1 again:

$$
\begin{aligned}
\cdots \underbrace{C \cdots \dot{C}}_{c_{i-1} \geq 3} \underbrace{D}_{d_{i}=1} \underbrace{\dot{C} \cdots C}_{c_{i} \geq 3} \cdots & \stackrel{0}{\rightarrow} \cdots \underbrace{C \cdots C}_{c_{i-1}^{\prime} \geq 3} \underbrace{\dot{D} D}_{d_{i}^{\prime}=3} \underbrace{\dot{D} \cdots C}_{c_{i}^{\prime} \geq 3} \cdots \\
& \stackrel{0}{\rightarrow} \cdots \underbrace{C \cdots \dot{C}}_{c_{i-1}^{\prime \prime} \geq 3} \underbrace{D}_{d_{i}^{\prime \prime}=1} \underbrace{\dot{C} \cdots C}_{c_{i}^{\prime \prime} \geq 3} \cdots .
\end{aligned}
$$

Hence, $d$ can be only 1,2 , or 3 .

(S4) Equation (3.3) holds for any state $s \in M$.

Suppose that $s \in M$ is as shown in (3.2) with $d_{i}=d_{i+1}=1$. By (5.7) and (5.15),

$$
\begin{aligned}
& \cdots \underbrace{C \cdots C}_{c_{i-1}} \underbrace{\stackrel{\bullet}{D}}_{d_{i}=1} \underbrace{C \cdots C}_{c_{i}} \underbrace{\dot{D}}_{d_{i+1}=1} \underbrace{C \cdots C}_{c_{i+1}} \cdots \\
& \quad \stackrel{0}{\rightarrow} \cdots \underbrace{C \cdots C}_{c_{i-1}^{\prime}} \underbrace{D \dot{D} D}_{d_{i}^{\prime}=3} \underbrace{C \cdots C}_{c_{i}-2} \underbrace{D \dot{D} D}_{d_{i+1}^{\prime}=3} \underbrace{C \cdots C}_{c_{i+1}^{\prime}} \cdots \\
& \quad=\boldsymbol{u} .
\end{aligned}
$$

Since $\boldsymbol{u} \in M$ by (S1), we have $c_{i}-2 \geq 3$ by (S2). That is, $c_{i} \geq 5$. This verifies (3.3) for $\left(d_{i}, d_{i+1}\right)=(1,1)$. The other cases can be treated similarly and the details are thus omitted.

(S5) The following diagram shows $M$ is indeed what we claimed:

$$
\begin{aligned}
& \cdots \underbrace{D D}_{2} \underbrace{C \cdots C}_{c_{i-1} \geq 4} \underbrace{\dot{D}}_{1} \underbrace{C \cdots C}_{c_{i} \geq 3} \underbrace{D \dot{D} D}_{3} \underbrace{C \cdots C}_{c_{i+1}} \cdots \\
& \stackrel{\leftrightarrow}{\leftrightarrow} \cdots \underbrace{D D}_{2} \underbrace{C \cdots C}_{c_{i-1}-1} \underbrace{D D D}_{3} \underbrace{C \cdots C}_{c_{i}} \underbrace{\stackrel{D}{D}}_{1} \underbrace{C \cdots C}_{c_{i+1}^{\prime}} \cdots .
\end{aligned}
$$

So the length $d$ of any $D$-string in $s \in M$ obeys the following rule under $Q_{0}$ :

$$
1 \rightarrow 3, \quad 3 \rightarrow 1, \quad \text { and } \quad 2 \rightarrow 2
$$

Therefore, the closed connected component $F(s)$ containing $s \in M$ has cardinal number 2 or 1 depending on whether some $d_{i} \in\{1,3\}$ in its representation (3.2). In case $|F(s)|=2$, we denote the other element by $s_{d}$. So $s \stackrel{0}{\leftrightarrow} s_{d}$.

Some consequences from the arguments above are listed below.

(S6) Any $D$-string in a state $s \in S$ can never be eliminated under $Q_{0}$. 
Unless $\boldsymbol{s}$ goes to $\boldsymbol{D}$, the length of any D-string in $\boldsymbol{s}$ will become 1, 2, or 3 under $Q_{0}$.

(S7) Any $C$-string with length greater than or equal to 5 in a state $s \in S$ can never be eliminated under $Q_{0}$.

If not increased, its length could decrease at most by 2 under $Q_{0}$.

(S8) Let $s \in M$ be as given in (3.2). For any $i$,

$$
d_{i}+c_{i}+d_{i+1}+c_{i+1} \geq 10
$$

and the equality holds if and only if $\left(d_{i}, c_{i}, d_{i+1}, c_{i+1}\right)$ is an element in the following set:

$$
\{(2,3,2,3),(1,3,3,3),(3,3,1,3),(1,5,1,3),(1,4,2,3),(2,4,1,3)\} .
$$

Based on (3.3), both claims can be easily verified case by case as $d_{i}, d_{i+1} \in\{1,2,3\}$.

By (5.31), the minimum total length of two adjacent pairs of $D$-strings and $C$-strings is 10 . As in (5.19), we decompose $M$ as $M=\bigcup_{k=1}^{L} M_{k}$, where $L=\lfloor n / 5\rfloor$. The structure of $M_{L}$ for $5 \mid n$, which plays a special role in the theorem, is determined as follows.

(S9) If $5 \mid n$ and $L$ is odd, then $M_{L}=M_{2,3}$.

Let $s \in M_{L}$ be as given in (3.2). We need to show that $\left(d_{i}, c_{i}, d_{i+1}, c_{i+1}\right)=(2,3,2,3)$ for any $i$. Suppose that $\left(d_{i}, c_{i}, d_{i+1}, c_{i+1}\right) \neq(2,3,2,3)$ for any $i$. It is clear from (5.32) that we have either $d_{i}+c_{i} \geq 6$ or $d_{i+1}+c_{i+1} \geq 6$ unless $\left(d_{i}, c_{i}, d_{i+1}, c_{i+1}\right)=(1,4,2,3)$. In this exceptional case, from (3.3) we obtain $\left(d_{i-1}, c_{i-1}\right) \geq(1,5),(2,4)$, or $(3,3)$. Hence, $d_{i-1}+c_{i-1} \geq 6$. Putting together, we may conclude that

$$
d_{j}+c_{j} \geq 6 \text { for some } j .
$$

By (5.31), $d_{j+1+2 k}+c_{j+1+2 k}+d_{j+2+2 k}+c_{j+2+2 k} \geq 10$ for each $0 \leq k \leq(L-1) / 2$. Because $L$ is odd, by adding up over $k$ and using (5.33), we obtain $n=|s| \geq 6+10(L-1) / 2=5 L+1=$ $n+1$, which is a contradiction. This verifies the claim.

(S10) If $5 \mid n$ and $L$ is even, then any $s \in M_{L}$ is either in $M_{2,3}$ or has the form

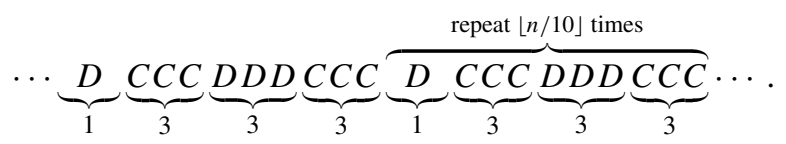

Because the equality in (5.31) holds for any $i$ in representation (3.2) for $s \in M_{L},\left(d_{i}, c_{i}\right.$, $\left.d_{i+1}, c_{i+1}\right)$ belongs to the set in (5.32). Suppose that $s \notin M_{2,3}$. If some $\left(d_{i}, c_{i}\right)=(1,3)$ then $\left(d_{i+1}, c_{i+1}\right)$ can only be $(3,3)$ in order to be in $(5.32)$. This forces $\left(d_{i+2}, c_{i+2}\right)=(1,3)$ in turn by (5.32). Repeating the same arguments, we are led to the form in (5.34). Only the case in which $\left(d_{i}, c_{i}, d_{i+1}, c_{i+1}\right)=(1,4,2,3)$ is left from (5.32). As in (S9), we then have $\left(d_{i-1}, c_{i-1}\right) \geq(1,5),(2,4)$, or $(3,3)$. Hence, $d_{i-1}+c_{i-1}+d_{i}+c_{i} \geq 11$, which violates the equality in (5.31).

Now we are ready to find $S_{*}$. In view of the statements in Theorem 3.2(ii), part (ii) is much more complicated to prove than part (i). Though still valid, (5.6) is inadequate for our purpose. 
For any $\boldsymbol{s}, \boldsymbol{u} \in S_{0}$, we need to find a minimum-cost path $\gamma=\left\{\boldsymbol{s}_{0}=\boldsymbol{s}, \boldsymbol{s}_{1}, \ldots, \boldsymbol{s}_{\ell}=\boldsymbol{u}\right\}$ from $\boldsymbol{s}$ to $\boldsymbol{u}$. We will show in the following steps that

$$
\text { there exists a } \gamma \text { with each } U\left(s_{i}, s_{i+1}\right)= \begin{cases}1 & \text { if } s_{i} \in S_{0} \text { and } s_{i+1} \notin F\left(s_{i}\right), \\ 0 & \text { otherwise }\end{cases}
$$

first for $\boldsymbol{s}, \boldsymbol{u}$ in some $M_{k}$ and then for states in $\{\boldsymbol{C}\} \cup M \backslash M_{2,3}$. Finally, we will show how $\boldsymbol{D}$ communicates with other states. Note that the path $\gamma$ is certainly optimal in view of (5.1) and (5.5).

Step 1. Statement (5.35) holds for any $\boldsymbol{s}, \boldsymbol{u} \in M_{k}$ under the condition that

$$
5 \nmid n \quad \text { or } \quad 1 \leq k<L:=\left\lfloor\frac{n}{5}\right\rfloor .
$$

Moreover, the path $\gamma$ in (5.35) lies entirely in $M_{k}$ and is reversible. Roughly speaking, the above means that all states in $M_{k}$ are 'equivalent' to each other under condition (5.36).

Because the path $\gamma$ above is required to be reversible, it suffices to verify the claim for any $\boldsymbol{u} \in M_{k} \cap M_{2, \geq 3}$. That is, all its $D$-strings have length 2 . As will now be shown, this can be further weakened. By (5.36) and (S9)-(S10), there are at least $\min (5, n-5 L) \geq 1$ extra $C$ s in $\boldsymbol{u}$. Hence, some $c_{i}^{\prime} \geq 4$ in representation (3.2) for $\boldsymbol{u}$. Let $\boldsymbol{v}, \boldsymbol{w} \in M_{k}$ be exactly as $\boldsymbol{u}$ except that the lengths from the $i$ th $D$-string to the $(i+1)$ th $C$-string are $\left(2, c_{i}^{\prime}, 1, c_{i+1}^{\prime}+1\right)$ and $\left(2, c_{i}^{\prime}-1,2, c_{i+1}^{\prime}+1\right)$, respectively. Then

$$
\boldsymbol{u} \stackrel{1}{\leftrightarrow} \boldsymbol{v} \stackrel{0}{\leftrightarrow} \boldsymbol{v}_{d} \stackrel{0}{\leftrightarrow} \boldsymbol{v} \stackrel{1}{\leftrightarrow} \boldsymbol{w}, \quad \text { where } \quad \boldsymbol{v}_{d} \in F(\boldsymbol{v}) .
$$

This means that we can shift any extra $C$ in a $C$-string of $\boldsymbol{u}$ to its right $C$-string while fulfilling the claim. By symmetry, this extra $C$ can also be shifted to its left $C$-string. Repeating the same procedures, all the extra $C$ s in $\boldsymbol{u}$ can be arbitrarily relocated among its $k C$-strings, while all encountered states are in $M_{k}$ and fulfill the claim. Hence, it suffices to verify the claim for some $\boldsymbol{u} \in M_{k} \cap M_{2, \geq 3}$.

Let $s \in M_{k}$ be as in (3.2). By (S3), there are nine possibilities for any $\left(d_{i}, d_{i+1}\right)$. By symmetry and (5.30), it suffices to consider the following two cases:

$$
\left(d_{i}, d_{i+1}\right) \in\{(1,1),(2,1),(2,2)\} \quad(\text { case }(\mathrm{a})), \quad\left(d_{i}, d_{i+1}\right)=(1,3) \quad(\text { case }(\mathrm{b})) .
$$

Let us start with case (a). Suppose that some $\left(d_{i}, d_{i+1}\right)=(1,1)$ in $(3.2)$. Observe that

$$
\begin{aligned}
\boldsymbol{s} & =\cdots C \underbrace{\stackrel{\bullet}{D}}_{1} \underbrace{C \cdots C}_{c_{i} \geq 5} \underbrace{\dot{D}}_{1} \cdots \\
& \stackrel{0}{\leftrightarrow} \cdots \underbrace{D \dot{D} \dot{D} D}_{3} \underbrace{C \cdots C}_{c_{i}-2 \geq 3} \underbrace{D \dot{D} D}_{3} \cdots \\
& \stackrel{1}{\leftrightarrow} \cdots \underbrace{*}_{2} \underbrace{\dot{D} D}_{c_{i}-1 \geq 4} \underbrace{C \cdots C}_{1} \underbrace{\dot{D}}_{1} \cdots \\
& =\boldsymbol{v} .
\end{aligned}
$$

Not only does $v \in M_{k}$ by (3.3) but also the size $d_{j}^{\prime}$ of its $j$ th $D$-string equals $d_{j}$ for any $1 \leq j \leq k$ except $d_{i}^{\prime}=2$. Since the path above is reversible, the claim in step 1 holds for $s$ 
and $\boldsymbol{u}=\boldsymbol{v}$. Therefore, we may assume without loss of generality that some $d_{i}=2$ in (3.2) for $\boldsymbol{s}$ satisfying case (a) in (5.37).

If $s \notin M_{2, \geq 3}$ then, by (5.30), we may assume some $\left(d_{i}, d_{i+1}\right)=(2,1)$ in (3.2) for $s$. Then $c_{i} \geq 4$ by (3.3). As above, we have

$$
\begin{aligned}
\cdots \underbrace{D D}_{2} \underbrace{C \cdots C}_{c_{i} \geq 4} \underbrace{\stackrel{D}{D}}_{1} \underbrace{C \cdots C}_{c_{i+1}} \cdots & \stackrel{0}{\leftrightarrow} \cdots \underbrace{D D}_{2} \underbrace{C \cdots C}_{c_{i}-1} \underbrace{\stackrel{\circ}{D} \dot{D} D}_{3} \underbrace{C \cdots C}_{c_{i+1}^{\prime}} \cdots \\
& \stackrel{\leftrightarrow}{\leftrightarrow} \cdots \underbrace{D D}_{2} \underbrace{C \cdots C}_{c_{i}-1 \geq 3} \underbrace{D \dot{D}}_{2} \underbrace{\stackrel{*}{C} \cdots C}_{c_{i+1}} \cdots \\
& =w,
\end{aligned}
$$

where $w \in M_{k}$ and the size $d_{j}^{\prime}$ of its $j$ th $D$-string equals $d_{j}$ for any $1 \leq j \leq k$ except $d_{i+1}^{\prime}=2$. Repeating the same procedures, we will find a reversible path from $\boldsymbol{s}$ to some $\boldsymbol{u} \in M_{k} \cap M_{2, \geq 3}$ as claimed.

By (5.37), it remains to consider the case in which $\left(d_{i}, d_{i+1}\right) \in\{(1,3),(3,1)\}$ for all $i$. Because the case in which some $\left(d_{i}, d_{i+1}\right)=(1,1)$ or $(3,3)$ has just been treated above, we may assume without loss of generality that $\left(d_{1}, d_{2}, d_{3}, \ldots, d_{k}\right)=(1,3,1,3, \ldots, 1,3)$. In particular, $k$ is even. If all $c_{i}=3$ in (3.2) for $s$ then $k=L$ by (5.34), which is excluded by (5.36). Hence, some $c_{i} \geq 4$. By symmetry we may assume that $\left(d_{i}, d_{i+1}\right)=(1,3)$. Then

$$
\begin{aligned}
\boldsymbol{s}_{d} & \stackrel{0}{\leftrightarrow} \cdots \underbrace{D \dot{D} D}_{3} \underbrace{C \cdots \stackrel{\circ}{C}}_{c_{i-1} \geq 3} \underbrace{\stackrel{\bullet}{D}}_{1} \underbrace{C \cdots C}_{c_{i} \geq 4} \underbrace{D \dot{D} D}_{3} \cdots \\
& \stackrel{1}{\leftrightarrow} \cdots \underbrace{\stackrel{\bullet}{D}}_{1} \underbrace{C \cdots C}_{c_{i-1}+1 \geq 4} \underbrace{\stackrel{D}{D}}_{2} \underbrace{C \cdots C}_{c_{i} \geq 4} \underbrace{\stackrel{D}{D} \cdots}_{1} \cdots \\
& =\boldsymbol{m} .
\end{aligned}
$$

By (3.3), $\boldsymbol{m} \in M_{k}$ with the length of its $i$ th $D$-string being 2. Hence, $\boldsymbol{m}$ satisfies case (a). We have just shown above that there is a desired, reversible path from $\boldsymbol{m}$ to some $\boldsymbol{u} \in M_{k} \cap M_{2, \geq 3}$. Putting together, this completes the proof of step 1 .

Step 2. Let $M_{0}=\{\boldsymbol{C}\}$. For $n \geq 6$ and $1 \leq k \leq L$, (5.35) holds for any $\boldsymbol{s} \in M_{k} \backslash M_{2,3}$ and $\boldsymbol{u} \in M_{k-1}$. Moreover, (5.35) holds for $\boldsymbol{u} \in M_{k} \backslash M_{2,3}$ and $\boldsymbol{s} \in M_{k-1}$ as well.

Note that $M_{2,3} \subseteq M_{L}$ and $M_{2,3} \neq \varnothing$ if and only if $5 \mid n$. By step 1, it suffices to consider some $\boldsymbol{u} \in M_{k-1}$. We first treat the case in which (5.36) holds. By step 1 again we may assume that all $d_{j}=2$ except a certain $d_{i}=1$ in representation (3.2) for $\boldsymbol{s}$. Note that such $\boldsymbol{s}$ exist under (5.36). So both $c_{i-1}, c_{i} \geq 4$ by (3.3). The conclusion is implied by the following diagram:

$$
\boldsymbol{s}=\cdots \underbrace{D D}_{2} \underbrace{C \cdots C}_{c_{i-1}} \underbrace{\dot{D}}_{1} \underbrace{C \cdots C}_{c_{i}} \underbrace{D D}_{2} \cdots \stackrel{0}{\leftrightarrow} \boldsymbol{s}_{d} \stackrel{1}{\rightarrow} \cdots \underbrace{D D}_{2} \underbrace{C \cdot \dot{C} \cdot C}_{c_{i-1}+1+c_{i}} \underbrace{D D}_{2} \cdots=\boldsymbol{u} \stackrel{1}{\rightarrow} \boldsymbol{s} .
$$

Note that $\boldsymbol{u} \in M_{k-1}$ by (3.3) and the path above is nearly reversible in the sense that $\boldsymbol{s}_{d}$ appears only in the path from $\boldsymbol{s}$ to $\boldsymbol{u}$.

It remains to consider the case in which $5 \mid n$ and $k=L=n / 5$. By (S9)-(S10), any $s \in M_{L} \backslash M_{2,3}$ has the form shown in (5.34). A diagram similar to (5.38) works for such $s$.

Step 3. For $n \geq 6$, (5.35) holds for any $\boldsymbol{s}, \boldsymbol{u} \in\left(S_{0} \backslash M_{2,3}\right) \backslash\{\boldsymbol{D}\}$. So all states in $\left(S_{0} \backslash\right.$ $\left.M_{2,3}\right) \backslash\{\boldsymbol{D}\}$ are 'equivalent'. 
By varying $k$ from $L$ to 1 in step 2, the conclusion follows. Now we consider $M_{2,3}$.

Step 4. For $n \geq 6$, (5.35) holds for any $s \in M_{2,3}$ and $\boldsymbol{u} \in M_{L-1}$. But the minimum cost to reach $M_{2,3}$ from $S_{0} \backslash M_{2,3}$ is 2 instead of 1 .

Since $n \geq 6$ and $M_{2,3} \neq \varnothing$ if and only if $5 \mid n$, we have $L=n / 5 \geq 2$. Then, by (5.15) and (5.27),

$$
\begin{aligned}
\cdots \underbrace{C C C}_{3} \underbrace{D D}_{2} \underbrace{C \dot{C} C}_{3} \underbrace{D D}_{2} \underbrace{C C C}_{3} \cdots & \rightarrow \cdots \underbrace{C C C}_{3} \underbrace{D D}_{2} C \dot{D} C \underbrace{D D}_{2} \underbrace{C C C}_{3} \cdots \\
& \stackrel{0}{\rightarrow} \cdots \underbrace{C \cdots C}_{3} \underbrace{D \cdots D}_{7} \underbrace{C \cdots C}_{3} \cdots \\
& \stackrel{0}{\rightarrow} \cdots \underbrace{C \cdots C}_{4} \underbrace{D \cdots D}_{5} \underbrace{C \cdots C}_{4} \cdots \\
& \stackrel{0}{\rightarrow} \cdots \underbrace{C \cdots C}_{5} \underbrace{D \dot{D} D}_{3} \underbrace{C \cdots C}_{5} \cdots \\
& \stackrel{0}{\leftrightarrow} \cdots \underbrace{C \cdots C}_{6} \underbrace{\dot{D}_{D}^{C}}_{1} \underbrace{C \cdots C}_{6} \cdots .
\end{aligned}
$$

Since the three intermediate states on the right-hand side are transient and the last two states are in $M_{L-1}$ with all their not shown $D$-strings and $C$-strings having lengths 2 and 3, respectively, the first claim is verified. Owing to step 3 and (5.30), the minimum cost to reach any $s \in M_{2,3}$ from $S_{0} \backslash M_{2,3}$ is

$$
M_{L-1} \ni \boldsymbol{u}=\cdots \underbrace{D \dot{D}}_{2} \underbrace{C \cdots C}_{8} \underbrace{\dot{D} D}_{2} \cdots \stackrel{2}{\rightarrow} \cdots \underbrace{D \dot{D}}_{2} \underbrace{C C C}_{3} \underbrace{D D}_{2} \underbrace{C C C}_{3} \underbrace{\dot{D} D}_{2} \cdots
$$

It remains to consider how $\boldsymbol{D}$ communicates with other states. The cases in which $n=5$ and $n \geq 6$ will be treated separately in the following two steps.

Step 5. Consider the case in which (3.4)-(3.8) hold for $n=5$. In this case, $L=\lfloor n / 5\rfloor=1$ and $M=M_{1}=M_{2,3}$. Different from (5.39), we simply have

$$
D D C \stackrel{\circ}{C} C \stackrel{1}{\rightarrow} D D C D C \stackrel{0}{\rightarrow} D \stackrel{3}{\rightarrow} D D \stackrel{\circ}{C} \stackrel{\circ}{C} \stackrel{\circ}{C} \in M_{1} .
$$

The last move is optimal as we have shown in (S2) that any $C$-string with length less than or equal to 2 in a state will be eliminated under $Q_{0}$, which implies that $\boldsymbol{D}$ cannot reach any other state in $S_{0}$ at cost less than or equal to 2. By using (S6), (5.15), and (5.27), the optimal path to reach in and out of $\boldsymbol{C}$ is as follows:

$$
M_{1} \ni \stackrel{\circ}{D} \stackrel{\circ}{D} C C C \stackrel{2}{\rightarrow} C \stackrel{1}{\rightarrow} C C \stackrel{\bullet}{D} C C \stackrel{0}{\rightarrow} C D \dot{D} D C \stackrel{0}{\rightarrow} D C \dot{D} C D \stackrel{0}{\rightarrow} D .
$$

Since $M=M_{2,3}$ and $\left|M_{2,3}\right|=5$, we summarize (5.40) and (5.41) to obtain

$$
v(\{\boldsymbol{D}\})=6, \quad v(\{\boldsymbol{C}\})=9, \quad \text { and } \quad v(\{\boldsymbol{s}\})=8 \quad \text { for } \boldsymbol{s} \in M_{1} .
$$

Hence, $v(\{\boldsymbol{D}\})=v_{1}=6, S_{*}=\{\boldsymbol{D}\}$, and $\mu_{\varepsilon}(\boldsymbol{C}) \approx \varepsilon^{3}$ by (4.6). Moreover, $v(\{\boldsymbol{C}, \boldsymbol{D}\})=$ $v_{2}=5$. Then $k_{0}=2$ and $\delta=v_{1}-v_{2}=1$ in (4.8). This completes step 5 . 
Step 6. Consider the case in which (3.4)-(3.8) hold for $n \geq 6$. By using (5.15) and (5.27), $D$ can reach out at the minimum cost 3 , as in (5.40):

$$
\boldsymbol{D} \stackrel{3}{\rightarrow} \underbrace{\stackrel{\circ}{C} \stackrel{\circ}{C}}_{3} \underbrace{D \cdots D}_{n-3} \stackrel{0}{\rightarrow} \underbrace{C \cdots C}_{5} \underbrace{D \cdots D}_{n-5} \stackrel{0}{\rightarrow} \underbrace{C \cdots C}_{7} \underbrace{D \cdots D}_{n-7} \stackrel{0}{\rightarrow} \cdots \stackrel{0}{\rightarrow} \boldsymbol{u} \in M_{1},
$$

where the unique $D$-string in $\boldsymbol{u}$ has length 1 or 2 depending on whether $n$ is even or odd, respectively. Let $\eta$ be the number of closed connected components in $M$. Together with steps 3 and 4,

$$
v(\{\boldsymbol{s}\})=3+\eta \quad \text { and } \quad v(\{\boldsymbol{u}\})=4+\eta \quad \text { for any } \boldsymbol{s} \in\{\boldsymbol{C}\} \cup M \backslash M_{2,3} \text { and } \boldsymbol{u} \in M_{2,3} .
$$

In order to find the minimum-cost path from $\boldsymbol{C}$ to $\boldsymbol{D}$, step 3 implies that it suffices to do so from any $\boldsymbol{s} \in\{\boldsymbol{C}\} \cup M \backslash M_{2,3}$. By (S6), it saves to use some state with as many $D$ s as possible. Note that any $D$-string in $s \in M$ has length less than or equal to 3 by (S3). If $d_{i}=d_{i+1}=1$ and $c_{i} \leq 9$ in representation (3.2) for $s \in M$, the following diagram indicates that the $i$ th $C$-string of $s$ can be eliminated at cost 1 :

$$
\begin{aligned}
& \boldsymbol{s} \stackrel{0}{\leftrightarrow} s_{d} \\
& \stackrel{1}{\rightarrow} \cdots * \underbrace{\stackrel{\circ}{D}}_{1} \underbrace{C C C C}_{4} \underbrace{\stackrel{\circ}{D}}_{1} \underbrace{C C C C}_{4} \underbrace{\stackrel{\bullet}{D}}_{1} * \cdots \\
& \stackrel{0}{\rightarrow} \cdots \underbrace{D \dot{D} D}_{3} \underbrace{C C}_{2} \underbrace{D D D}_{3} \underbrace{C C}_{2} \underbrace{D \dot{D} D}_{3} \cdots \\
& \stackrel{0}{\rightarrow} \cdots * \underbrace{\stackrel{\bullet}{D}}_{1} C D D C D C D D C \underbrace{\stackrel{\bullet}{D}}_{1} * \cdots \\
& \stackrel{0}{\rightarrow} \cdots \underbrace{D \dot{D} D \cdots D \dot{D} D}_{13} \cdots .
\end{aligned}
$$

At least two mutations in $s_{d}$ are needed to eliminate this $C$-string of $s$ if $c_{i}>9$ and at least three mutations if $c_{i}>14$. The reason is as follows. If we make two such mutations in the $i$ th $C$-string of $\boldsymbol{s}_{d}$, they need to be well separated. Then the resulting state $\boldsymbol{u}$ under $Q_{0}$ is the same as $s$, except the original $i$ th $C$-string of $s$ is now divided into three shorter $C$-strings with lengths $c_{i 1}, c_{i 2}$, and $c_{i 3}$, respectively. By (5.7) and (5.15), each of these three $C$-strings will shrink by 2 under $Q_{0}$ at the next period, while their four surrounding $D$-strings grow from length 1 to length 3. If $c_{i}>14$ then $c_{i j}-2 \geq 3$ for some $j$. By (5.15) and (5.27), this newly created $C$-string will grow back to length $c_{i j} \geq 5$ in the following period and can never be eliminated by (S7). In general, if

$$
c_{i}>\ell+2(\ell+1)+2(\ell+1)=5 \ell+4
$$

then at least $(\ell+1)$ mutations in the $i$ th $C$-string of $s_{d}$ are needed in order to eliminate the $i$ th $C$-string of $s$ under $Q_{0}$. Otherwise, $\ell$ mutations are enough, as in (5.43). Since the increment from $\ell$ to $(\ell+1)$ is $(5(\ell+1)+4)-(5 \ell+4)=5$, it saves to have in $s$ as many blocks of $D C C C C C C C C C$ as possible, as one mutation is enough to eliminate the nine $C$ s in any such block. The maximum number allowed for such blocks in $s$ is $\lfloor n / 10\rfloor$. If the remainder is $r=n-10\lfloor n / 10\rfloor \geq 6$, we may add in an extra $D C \cdots C$ block with one $D$ and $r-1 \geq 5 C$ s in order to make $s \in M \backslash M_{2,3}$. If $r \leq 5$, we may insert $r C$ s in some $C$-string. So $s \in M \backslash M_{2,3}$ 
has the form

$$
\overbrace{1}^{\overbrace{D}^{\text {repeat }\lfloor n / 10\rfloor \text { times }} \underbrace{C C C C C C C C C}_{9}} \cdots \underbrace{D}_{1} \underbrace{C \cdots C}_{r-1} \quad \text { or } \overbrace{1}^{\overbrace{D}^{\text {repeat }\lfloor n / 10\rfloor-1 \text { times }} \underbrace{C C C C C C C C C C}_{9}} \cdots \underbrace{D}_{1} \underbrace{C \cdots C}_{9+r} .
$$

In either case, one more mutation from $C$ to $D$ is enough by (5.44) to eliminate these extra $C$ s. Hence, with $s$ given in (5.45), we have, from (5.43),

$$
s \stackrel{0}{\rightarrow} s_{d} \stackrel{\lceil n / 10\rceil}{\rightarrow} u_{1} \stackrel{0}{\rightarrow} u_{2} \stackrel{0}{\rightarrow} u_{3} \stackrel{0}{\rightarrow} D
$$

where all the $\boldsymbol{u}_{i}$ are transient states, as in (5.43). In fact, this is also the most economic way to reach $\boldsymbol{D}$ from $M$. This is because $n / 5 \geq\lceil n / 10\rceil$ for $5 \mid n$ and any state in $M_{2,3}$, which is nonempty if and only if $5 \mid n$, has $n / 5 C$-strings. One mutation is needed for eliminating any of the $C$-strings.

It follows, from steps 3 and 4 and (5.46), that

$$
v(\{\boldsymbol{D}\})=\left\lceil\frac{n}{10}\right\rceil+\eta .
$$

Combining (5.47) with (5.42), we obtain $v_{1}=\min (3,\lceil n / 10\rceil)+\eta$. By using (4.6)-(4.8), (3.4)-(3.8) for $n \geq 6$ follow easily. For example, $\lceil n / 10\rceil=3$ for $21 \leq n \leq 30$. By (4.6), (5.42), and (5.47), $S_{*}=S_{0}$ for $21 \leq n<30$ but $n \neq 25$ and $S_{*}=S_{0} \backslash M_{2,3}$ for $n=25$ or 30. In the former case, $\mathrm{E}_{\varepsilon}(T) \approx \varepsilon^{0}$ by (5.2). In the latter case, $v_{2}=v(\{\boldsymbol{s}, \boldsymbol{u}\})=2+\eta$ for any $\boldsymbol{s} \in S_{0} \backslash M_{2,3}$ and $\boldsymbol{u} \in M_{2,3}$. Hence, $\delta=v_{1}-v_{2}=1$ and $\mathrm{E}_{\varepsilon}(T) \approx \varepsilon^{-1}$ by (4.8). The other cases of $n$ can be easily checked. It is interesting to note that, for $n \geq 31, v_{1}=3+\eta<v(\{\boldsymbol{D}\})$, $v_{2}=\eta=v(\{\boldsymbol{D}, \boldsymbol{C}\})$, and $\delta=v_{1}-v_{2}=3$. Hence, $S_{*}=\left(S_{0} \backslash M_{2,3}\right) \backslash\{\boldsymbol{D}\}$ and $\mathrm{E}_{\varepsilon}(T) \approx \varepsilon^{-3}$. In particular, $\boldsymbol{C} \in S_{*}$ but $\boldsymbol{D} \notin S_{*}$. The proof of part (ii) is completed.

Part (iii): $a+b \leq(c+3 d) / 2$ and $(3 a+b) / 2 \geq c+d$. Now (5.15) remains true from the results in Table 4 in Appendix A and we have (5.11) from the results in Table 3 in Appendix A. Since all the configuration rules for the present case are exactly the same as in Theorem 3.1(ii), we naturally get the same conclusions as there. The details are therefore omitted.

\section{Appendix A}

In Tables 1, 2, 3, and 4 the state column depicts the strategies adopted by five consecutive players $i-2, i-1, i, i+1$, and $i+2$. The payoff columns describe the total payoffs of players $i-1, i$, and $i+1$ under the imitation of the most successful player dynamics, and the average payoffs for strategies $D$ and $C$ under the imitation of the most successful action dynamics.

TABLe 1.

\begin{tabular}{cccccc}
\hline \multirow{2}{*}{ State $\boldsymbol{s}$} & \multicolumn{5}{c}{ Payoffs } \\
\cline { 2 - 6 } & $z_{i-1}(\boldsymbol{s})$ & $z_{i}(\boldsymbol{s})$ & $z_{i+1}(\boldsymbol{s})$ & $a_{i}^{D}(\boldsymbol{s})$ & $a_{i}^{C}(\boldsymbol{s})$ \\
\hline$\cdots D C D C D \cdots$ & $2 c$ & $2 b$ & $2 c$ & $2 b$ & $2 c$ \\
$\cdots D C D C C \cdots$ & $2 c$ & $2 b$ & $c+d$ & $2 b$ & $\frac{3 c+d}{2}$ \\
$\cdots C C D C C \cdots$ & $c+d$ & $2 b$ & $c+d$ & $2 b$ & $c+d$ \\
\hline
\end{tabular}


TABLe 2.

\begin{tabular}{cccccc}
\hline \multirow{2}{*}{ State $\boldsymbol{s}$} & \multicolumn{5}{c}{ Payoffs } \\
\cline { 2 - 6 } & $z_{i-1}(\boldsymbol{s})$ & $z_{i}(\boldsymbol{s})$ & $z_{i+1}(\boldsymbol{s})$ & $a_{i}^{D}(\boldsymbol{s})$ & $a_{i}^{C}(\boldsymbol{s})$ \\
\hline$\cdots D D C D D \cdots$ & $a+b$ & $2 c$ & $a+b$ & $a+b$ & $2 c$ \\
$\cdots D D C D C \cdots$ & $a+b$ & $2 c$ & $2 b$ & $\frac{a+3 b}{2}$ & $2 c$ \\
$\cdots C D C D C \cdots$ & $2 b$ & $2 c$ & $2 b$ & $2 b$ & $2 c$ \\
\hline
\end{tabular}

TABle 3.

\begin{tabular}{cccccc}
\hline \multirow{2}{*}{ State $\boldsymbol{s}$} & \multicolumn{5}{c}{ Payoffs } \\
\cline { 2 - 6 } & $z_{i-1}(\boldsymbol{s})$ & $z_{i}(\boldsymbol{s})$ & $z_{i+1}(\boldsymbol{s})$ & $a_{i}^{D}(\boldsymbol{s})$ & $a_{i}^{C}(\boldsymbol{s})$ \\
\hline$\cdots D D D C D \cdots$ & $2 a$ & $a+b$ & $2 c$ & $\frac{3 a+b}{2}$ & $2 c$ \\
$\cdots D D D C C \cdots$ & $2 a$ & $1+b$ & $c+d$ & $\frac{3 a+b}{2}$ & $c+d$ \\
$\cdots C D D C D \cdots$ & $a+b$ & $a+b$ & $2 c$ & $a+b$ & $2 c$ \\
$\cdots C D D C C \cdots$ & $a+b$ & $a+b$ & $c+d$ & $a+b$ & $c+d$ \\
\hline
\end{tabular}

TABLE 4.

\begin{tabular}{cccccc}
\hline \multirow{2}{*}{ State $\boldsymbol{s}$} & \multicolumn{5}{c}{ Payoffs } \\
\cline { 2 - 6 } & $z_{i-1}(\boldsymbol{s})$ & $z_{i}(\boldsymbol{s})$ & $z_{i+1}(\boldsymbol{s})$ & $a_{i}^{D}(\boldsymbol{s})$ & $a_{i}^{C}(\boldsymbol{s})$ \\
\hline$\cdots D D C C D \cdots$ & $a+b$ & $c+d$ & $c+d$ & $a+b$ & $c+d$ \\
$\cdots D D C C C \cdots$ & $a+b$ & $c+d$ & $2 d$ & $a+b$ & $\frac{c+3 d}{2}$ \\
$\cdots C D C C D \cdots$ & $2 b$ & $c+d$ & $c+d$ & $2 b$ & $c+d$ \\
$\cdots C D C C C \cdots$ & $2 b$ & $c+d$ & $2 d$ & $2 b$ & $\frac{c+3 d}{2}$ \\
\hline
\end{tabular}

\section{Acknowledgements}

The first author gratefully acknowledges funding from the National Science Council (project number NSC 95-2415-H-305-004). The second author gratefully acknowledges funding from the National Science Council in Taiwan. Valuable suggestions provided by the anonymous referee are also highly appreciated. Of course, all remaining errors are ours.

\section{References}

Bergstrom, T. C. and Stark, O. (1993). How altruism can prevail in an evolutionary environment. Amer. Econom. Rev. 83, 149-155.

Chen, H. C., Chow, Y. AND Wu, L. C. (2007a). Imitation, local interaction, and coordination: part I. Working paper, National Taipei University.

Chen, H. C., Chow, Y. And Wu, L. C. (2007b). Imitation, local interaction, and coordination: part II. Working paper, National Taipei University.

Chiang, T.-S. And Chow, Y. (1989). A limit theorem for a class of inhomogeneous Markov processes. Ann. Prob. 17, 1483-1502. 
ChIANG, T.-S. AND CHOw, Y. (2007). Ventcel optimal graphs, minimum cost spanning trees and asymptotic probabilities. Appl. Anal. Discrete Math. 1, 265-275.

Chow, Y. AND Wu, L. C. (2009). Evolutionary prisoner's dilemma games with local interaction, imitation and random matching. In preparation.

Ellison, G. (1993). Learning, local interaction, and coordination. Econometrica 61, 1047-1071.

Eshel, I., Samuelson, L. AND Shaked, A. (1998). Altruists, egoists, and hooligans in a local interaction model. Amer. Econom. Rev. 88, 157-179.

Freidlin, M. I. And Wentzell, A. D. (1984). Random Perturbations of Dynamical Systems. Springer, New York.

Geman, D. and Geman, S. (1984). Stochastic relaxation, Gibbs distributions and the Bayesian restoration of images. IEEE Trans. Pattern Anal. Mach. Intellig. 6, 721-741.

KANDORI, M., MaIlath, G. J. AND Rob, R. (1993). Learning, mutation, and long run equilibria in games. Econometrica 61, 29-56.

Karandikar, R., Mookherjee, D., Ray, D. and Vega-Redondo, F. (1998). Evolving aspirations and cooperation. J. Econom. Theory 80, 292-331.

Kirkpatrick, S., Gebatt, C. D., Jr. And Vecchi, M. P. (1983). Optimization by simulated annealing. Science 220, 671-680.

Miekisz, J. (2008). Evolutionary game theory and population dynamics. In Multiscale Problems in the Life Sciences: From Microscopic to Macroscopic (Lecture Notes Math. 1940), Springer, Berlin, pp. 269-316.

NowaK, M. A. AND May, R. M. (1992). Evolutionary games and spatial chaos. Nature 359, 826-829.

NowaK, M. A. ANd May, R. M. (1993). The spatial dilemmas of evolution. Internat. J. Bifur. Chaos Appl. Sci. Eng. 3, 35-78.

NowaK, M. A. AND Sigmund, K. (1995). Invasion dynamics of the finitely repeated prisoner's dilemma. Games Econom. Behavior 11, 364-390.

Nowa, M. A., Bonhoeffer, S. And May, R. M. (1994). More spatial games. Internat. J. Bifur. Chaos Appl. Sci. Eng. 4, 33-56.

Outkin, A. V. (2003). Cooperation and local interaction in Prisoners' Dilemma game. J. Econom. Behavior Organization 52, 481-503.

Palomino, F. and Vega-Redondo, F. (1999). Convergence of aspirations and partial cooperation in the prisoner's dilemma. Internat. J. Game Theory 28, 465-488.

Robson, A. J. ANd Vega-Redondo, F. (1996). Efficient equilibrium selection in evolutionary games with random matching. J. Econom. Theory 70, 65-92.

Vega-Redondo, F. (2003). Economics and the Theory of Games. Cambridge University Press.

Wiseman, T. and Yilankaya, O. (2001). Cooperation, secret handshakes, and imitation in the prisoner's dilemma. Games Econom. Behavior 37, 216-242. 